\title{
Review Article \\ Differential Forms in Lattice Field Theories: An Overview
}

\author{
F. L. Teixeira \\ ElectroScience Laboratory, Department of Electrical and Computer Engineering, The Ohio State University, \\ Columbus, $\mathrm{OH}$ 43212, USA \\ Correspondence should be addressed to F. L. Teixeira; teixeira@ece.osu.edu
}

Received 13 November 2012; Accepted 11 December 2012

Academic Editors: J. Banasiak, F. Sugino, and G. F. Torres del Castillo

Copyright @ 2013 F. L. Teixeira. This is an open access article distributed under the Creative Commons Attribution License, which permits unrestricted use, distribution, and reproduction in any medium, provided the original work is properly cited.

We provide an overview on the application of the exterior calculus of differential forms to the ab initio formulation of lattice field theories, with a focus on irregular or "random" lattices.

\section{Introduction}

The need to formulate field theories on a lattice (mesh, grid) arises from two main reasons, which may occur simultaneously or not. First, the lattice provides a natural "regularization" of divergences in lieu of renormalization techniques [1]. Such regularization does not need to be viewed as an ad hoc step, but instead as a natural consequence of assuming the field theory to be, at some fundamental level, an effective ("low"-energy) description [2]. Second, the lattice provides a direct route to compute, in a nonperturbative fashion, quantities of interest by numerical simulations. Nontrivial domains and complex boundary conditions can then be easily treated as well [3-6]. For these, the use of irregular ("random") lattices are often of interest to gain geometrical flexibility. Irregular lattices are also of interest as a means to provide a potentially faster convergence to the continuum limit, near-isotropic lattice dispersion properties, and better "conservation" of some (e.g., long-range translational and rotational) symmetries $[7,8]$. In some cases, irregular lattices are useful for universality tests as well $[9,10]$.

Lattice theories are typically developed by taking the counterpart continuum theory as starting point and then applying discretization techniques whereby derivatives are approximated by finite differences or some constraints are enforced on the functional space of admissible solutions to be spanned by a finite set of "basis" functions (e.g., "Galerkin methods" such as spectral elements and finite elements). These discretization strategies have proved very useful in many settings; however, they often produce difficulties in the case of irregular ("random") lattices. Among such difficulties are (i) numerical instabilities in marching-on-time algorithms (regardless of the time integration method used), (ii) convergence problems in algorithms relying on iterative linear solvers, and (iii) spurious ("ghost") modes and/or extraneous degrees of freedom. These problems often (but not always) appear associated with highly skewed or obtuse lattice elements, or at the boundary between heterogeneous (hybrid) lattices subcomponent, comprising overlapped domains or "mesh-stitching" interfaces, for example. Clearly, such difficulties put a constraint on the geometric flexibility that irregular lattices are intended for, and may require stringent (and computationally demanding) mesh quality controls. These difficulties also impact the ability to utilize "mesh refinement" strategies based on a priori error estimates. The reasons behind these difficulties can be traced to an inconsistent rendering of the differential calculus and degrees of freedom on the lattice. A rough classification of those inconsistencies is provided in Appendix B.

The objective of this paper is to present an overview on the application of the exterior calculus of differential forms to the $\mathrm{ab}$ initio formulation of field theories on irregular lattices [1129]. In the exterior calculus framework, the lattice is treated as a cell complex (in the parlance of algebraic topology [30]) instead of simply a collection of discrete points, and dynamic fields are represented by means of discrete differential forms (cochains) of various degrees $[28,31,32]$. This prescription provides a basis for developing a consistent "discrete calculus" on irregular lattices, and discrete analogues to partial differential equations that better adhere to the underlying physics. 
This topic intersects many disparate application areas. For concreteness, we employ classical electrodynamics as the standard example here; however, whenever particularly relevant to do so, we provide brief pointers to other field theories as well. Although some familiarity with the exterior calculus of differential forms is assumed [18, 19, 33-39], the discussion is mostly kept at a tutorial level. Finally, we stress that this is a review paper and no claim of originality is intended.

\section{Premetric Lattice Equations}

Let us denote the space of differential $p$-forms on a smooth connected manifold $\Omega$ as $\Lambda^{p}(\Omega)$. From a geometric perspective, a differential $p$-form $\alpha^{p} \in \Lambda^{p}(\Omega)$ can be viewed as an oriented $p$-dimensional density, or an object naturally associated with $p$-dimensional domains of integration $U_{p}$ such that the lattice contraction ("pairing") below:

$$
\left\langle U_{p}, \alpha^{p}\right\rangle \doteq \int_{U_{p}} \alpha^{p}
$$

gives a real number (in our context) for each choice of $U_{p}$ [22]. On a lattice $\mathscr{K}, U_{p}$ is restricted to be a union of elements from the finite set of $p$-dimensional $N_{p}$-oriented lattice elements, which we denote by $\Gamma_{p}(\mathscr{K})=\left\{\sigma_{p, i}, i=\right.$ $\left.1, \ldots, N_{p}\right\}$. These are collectively called " $p$-chains." In four dimensions for example, they correspond to the possible unions of elements from the set of vertices (nodes) $\sigma_{0}$, edges (links) $\sigma_{1}$, facets (plaquettes) $\sigma_{2}$, volume cells (voxels) $\sigma_{3}$, and hypervolume cells $\sigma_{4}$, for $p=1, \ldots, 4$, respectively. In the discrete setting, the degrees of freedom are reduced to the set of pairings (1) on each one of the lattice elements.

On the lattice, the pairing above can be understood as a map $\mathscr{R}^{p}: \Lambda^{p}(\Omega) \rightarrow \Gamma^{p}(\mathscr{K})$ such that

$$
\mathscr{R}^{p}\left(\alpha_{p}\right)=\left\langle\sigma_{p, i}, \alpha^{p}\right\rangle \doteq \int_{\sigma_{p, i}} \alpha^{p}
$$

defines its action on the basis of $p$-chains. Note that we use $\Gamma^{p}(\mathscr{K})$ to denote the space dual to $\Gamma_{p}(\mathscr{K})$, that is, the space $p$-cochains. The latter can be viewed as the space of "discrete differential forms." Because of this, and with some abuse of language, we use the terminology "differential forms" and "cochains" interchangeably to denote the same objects in what follows. The map $\mathscr{R}^{p}$ is called the de Rham map [22].

The basic differential operator of exterior calculus is the exterior derivative $d$, applicable to any number of dimensions. The discretization of $d$ on a general irregular lattice can be effected by a straightforward application of the generalized Stokes' theorem [22]:

$$
\int_{\sigma_{p+1}} d \alpha^{p}=\int_{\partial \sigma_{p+1}} \alpha^{p}
$$

with $p=0, \ldots, 3$ in $n=4$. In the above, $\partial$ is the boundary operator, which simply maps a $p$-dimensional lattice element to the set of $(p-1)$-dimensional lattice elements that comprise its boundary, preserving orientation. This theorem sets $\partial$ as the formal adjoint of $d$ in terms of the pairing given in (1), that is, $\left\langle\sigma_{p+1}, d \alpha^{p}\right\rangle=\left\langle\partial \sigma_{p+1}, \alpha^{p}\right\rangle$. Computationally, the boundary operator can be implemented by means of incidence matrices $[22,29,40]$ such that

$$
\partial \sigma_{p+1, i}=\sum_{j} C_{i j}^{p} \sigma_{p, j}
$$

where the indices $i$ and $j$ run over all $(p+1)$ - and $p$ dimensional lattice elements, respectively. The incidence matrix entries are such that $C_{i j}^{p} \in\{-1,0,1\}$ for all $p$, with sign determined by the relative orientation of lattice elements $i$ and $j$. The restriction to this set of integer values reflects the "metric-free" nature of the exterior derivative: only information about element connectivity, that is, the combinatorial aspects of the lattice, is involved here. It turns out that the metric is fully encoded by Hodge star operators, the discretization of which will be discussed further down below.

Using (3) and (4), one can write

$$
\int_{\sigma_{p+1, i}} d \alpha^{p}=\sum_{j} C_{i j}^{p} \int_{\sigma_{p, j}} \alpha^{p}
$$

for all $i$, so that the derivative operation is replaced by a proper sum over $j$. On the lattice, the nilpotency of the operators $\partial \circ$ $\partial=d \circ d=0$ [41] is recovered by the constraint [22]:

$$
\sum_{k} C_{i k}^{p+1} C_{k j}^{p}=0
$$

for all $i$ and $j$.

\section{Example: Lattice Electrodynamics}

We write Maxwell's equations in a four-dimensional Lorentzian manifold $\Omega$ as [34]:

$$
\begin{gathered}
d F=0, \\
d G=* \mathscr{J},
\end{gathered}
$$

where $d$ is the four-dimensional exterior derivative, $F$ and $G$ are the so-called Faraday and Maxwell 2-forms, respectively, and $* \mathscr{J}$ is the charge-current density 3 -form. The Hodge star operator $*$ is an isomorphism that maps $p$-forms to $(4-p)$ forms, and more generally $p$ forms to $(n-p)$ forms in a $n$ dimensional manifold, and, as mentioned before, depends on the metric of $\Omega[22,23,34,35,42-45]$. The above equations are complemented by the relation $G=* F$, which indicates that $F$ and $G$ are "Hodge duals" of each other.

3.1. Primal and Dual Lattices. Since $F$ and $G$ are 2 -forms, they should be discretized as 2-cochains residing on plaquettes (2chains) of the 4-dimensional lattice; however, it is important to recognize that these two forms are of different types: $F$ is an "ordinary" (or "nontwisted") differential form, whereas $G$ (as well as $* \mathscr{J}$ ) is a "twisted" (or "odd") differential form [46]. The basic difference here has to do with orientation: ordinary forms have internal orientation whereas twisted forms have 
external orientation $[20,22,46-48]$. These two types of orientations exhibit different symmetries under reflection, a distinction akin to that between proper (or polar) tensors and pseudo (or axial) tensors. Only twisted forms admit integration in nonorientable manifolds. These two types of forms are associated with two distinct "cell complexes" (lattices), each one inheriting the corresponding orientation: the ordinary form $F$ is associated with the set of plaquettes $\Gamma_{2}$ on the "ordinary cell complex" $\mathscr{K}$, thus belonging to $\Gamma^{2}(\mathscr{K})$, while the twisted forms $G$ and $* \mathscr{J}$ are associated with the set of plaquettes $\widetilde{\Gamma}_{2}$ on the "twisted cell complex" $\widetilde{\mathscr{K}}[22,27,48$, 49], thus belonging to $\Gamma^{2}(\widetilde{\mathscr{K}})$. Consequently, we also have two sets of incidence matrices $C_{i j}^{p}$ and $\widetilde{C}_{i j}^{p}$, one for each lattice. It is convenient to denote $\mathscr{K}$ as the "primal lattice" and $\widetilde{\mathscr{K}}$ as the "dual lattice" [22].

As detailed further below, these two lattices become intertwined by the Hodge duality $F=* G$. The need for dual lattices can also be motivated from a purely combinatorial standpoint (as a means to preserve key topological properties from the continuum theory) [24] or from a strictly computational standpoint (e.g., to provide higher-order convergence to the continuum) [50-52].

3.2. $3+1$ Theory. At this point, it is suitable to degeometrize time and treat it simply as a parameter. This corresponds to the majority of low-energy applications involving Maxwell's equations, in which one is interested in predicting the field evolution along different spatial slices for a given set of initial and boundary conditions. In this case, we still use the symbols $\mathscr{K}$ and $\widetilde{\mathscr{K}}$ for the primal and dual lattices, but they now refer to three-dimensional spatial lattices. Similarly, $\Omega$ now refers to a three-dimensional Euclidean manifold. In such a $3+1$ setting, one can decompose $F$ and $G$ as

$$
\begin{gathered}
F=E \wedge d t+B, \\
G=D-H \wedge d t,
\end{gathered}
$$

and the source density as

$$
* \mathscr{J}=-J \wedge d t+\rho,
$$

where $\wedge$ is the wedge product, $E$ and $H$ are the electric intensity and magnetic intensity 1 -forms on $\Gamma_{1}$ and $\widetilde{\Gamma}_{1}$, respectively, $D$ and $B$ are the electric flux and magnetic flux 2-forms on $\widetilde{\Gamma}_{2}$ and $\Gamma_{2}$, respectively, $J$ is the electric current density 2 form on $\widetilde{\Gamma}_{2}$, and $\rho$ is the electric charge density 3 -form on $\widetilde{\Gamma}_{3}$ (corresponding assignments for the $2+1$ and $1+1$ cases are provided in [32]). As a result, Maxwell's equations reduce to

$$
\begin{gathered}
d E=-\partial_{t} B, \\
d H=\partial_{t} D+J,
\end{gathered}
$$

representing Faraday's and Ampere's laws, respectively. Here, $d$ stands for the 3-dimensional spatial exterior derivative. Note that both (10) and (11) are metric-free. They are supplemented by Hodge star relations given by

$$
\begin{aligned}
& D=\star_{\epsilon} E, \\
& H=\star_{\mu^{-1}} B
\end{aligned}
$$

now involving two Hodge star maps in three-dimensional space: ${ }_{\epsilon}: \Lambda^{1}(\Omega) \rightarrow \Lambda^{2}(\Omega)$ and $\star_{\mu^{-1}}: \Lambda^{2}(\Omega) \rightarrow \Lambda^{1}(\Omega)$. On the lattice, we have the corresponding discrete counterparts: $\left[\star_{\epsilon}\right]: \Gamma^{1}(\mathscr{K}) \rightarrow \Gamma^{2}(\widetilde{\mathscr{K}})$ and $\left[\star_{\mu^{-1}}\right]: \Gamma^{2}(\mathscr{K}) \rightarrow \Gamma^{1}(\widetilde{\mathscr{K}})$. The subscripts $\epsilon$ and $\mu$ in $\star_{\epsilon}$ and $\star_{\mu^{-1}}$ serve to indicate that these operators also incorporate macroscopic constitutive material properties through the local permittivity and permeability values [53] (we assume dispersionless media for simplicity). In Riemannian manifolds (and in particular, Euclidean space) and reciprocal media, these two Hodge star operators are symmetric and positive-definite [54].

In what follows, we employ the following short-hand notation for cochains: $\left\langle\sigma_{1, i}, E\right\rangle=E_{i},\left\langle\widetilde{\sigma}_{1, i}, H\right\rangle=H_{i}$, $\left\langle\widetilde{\sigma}_{2, i}, D\right\rangle=D_{i},\left\langle\sigma_{2, i}, B\right\rangle=B_{i},\left\langle\widetilde{\sigma}_{2, i}, J\right\rangle=J_{i}$, and $\left\langle\widetilde{\sigma}_{3, i}, \rho\right\rangle=\rho_{i}$, where the indices run over the respective basis of $p$-chains in either $\mathscr{K}$ or $\widetilde{\mathscr{K}}, p=1,2,3$. With the exception of Appendix A, we restrict ourselves to the $3+1$ setting throughout the remainder of this paper.

\section{Casting the Metric on a Lattice}

4.1. Whitney Forms. The Whitney map $\mathscr{W}: \Gamma^{p}(\mathscr{K}) \rightarrow$ $\Lambda^{p}(\Omega)$ is the right-inverse of the de Rham map (2), that is, $\mathscr{R} \circ \mathscr{W}=\mathscr{I}$, where $\mathscr{I}$ is the identity operator. In simplicial lattices, this morphism can be constructed using the so-called Whitney forms [15, 22, 36, 43, 55-61] which are basic interpolants from cochains to differential forms [33] (other interpolants are also possible $[62,63]$ ). By definition, all cell elements of a simplicial lattice are simplices, that is, cells whose boundaries are the union of a minimal number of lower-dimensional cells. In other words, 0 -simplices are nodes, 1-simplices are links, 2-simplices are triangles, 3simplices are tetrahedra, and so on. Note that if the primal lattice is simplicial, the dual lattice is not [31]. For a $p$-simplex $\sigma_{p, i}$, the (lowest-order) Whitney form is given by

$$
\begin{aligned}
\omega^{p} & {\left[\sigma_{p, i}\right] } \\
\quad & \quad p ! \sum_{j=0}^{p}(-1)^{i} \lambda_{i, j} d \lambda_{i, 0} \wedge d \lambda_{i, 1} \cdots d \lambda_{i, j-1} \wedge d \lambda_{i, j+1} \cdots d \lambda_{i, p},
\end{aligned}
$$

where $\lambda_{i, j}, j=0, \ldots, p$, are the barycentric coordinates associated with $\sigma_{p, i}$. In the case of a 0 -simplex (node), (13) reduces to $\omega^{0}\left[\sigma_{0, i}\right]=\lambda_{i}$.

From its definition, it is clear that Whitney forms have compact support. Among its important structural properties are

$$
\left\langle\sigma_{p, i}, \omega^{p}\left[\sigma_{p, j}\right]\right\rangle=\int_{\sigma_{p, i}} \omega^{p}\left[\sigma_{p, j}\right]=\delta_{i j},
$$

where $\delta_{i j}$ is the Kronecker delta, which is simply a restatement of $\mathscr{R} \circ \mathscr{W}=\mathscr{I}$, and

$$
\omega^{p}\left[\partial^{T} \sigma_{p-1, i}\right]=d\left(\omega^{p-1}\left[\sigma_{p-1, i}\right]\right),
$$

where $\partial^{T}$ is the coboundary operator [56], consistent with the generalized Stokes' theorem. Further structural properties are 
provided in $[57,58]$. Higher-order version of Whitney forms also exist $[59,60]$. The key result $\mathscr{W} \circ \mathscr{R} \rightarrow \mathscr{I}$ holds in the limit of zero lattice spacing. This is discussed, together with other related convergence results in various contexts, in $[15$, 33, 64-68].

Using the short-hand $\omega^{p}\left[\sigma_{p, i}\right]=\omega_{i}^{p}$, we can write the following expansions for $E$ and $B$ in a irregular simplicial lattice, in terms of its cochain representations:

$$
\begin{aligned}
& E=\sum_{i} E_{i} \omega_{i}^{1}, \\
& B=\sum_{i} B_{i} \omega_{i}^{2},
\end{aligned}
$$

where the sums run over all primal lattice edges and faces, respectively.

One could argue that Whitney forms are continuum objects that should have no fundamental place on a truly discrete theory. In our view, this is only partially true. In many applications (see, e.g., the discussion on space-charge effects below), it is less natural to consider the lattice as endowed with some a priori discrete metric structure than it is to consider it instead as embedded in an underlying continuum (say, Euclidean) manifold with metric and hence inheriting all metric properties from it. In the latter case, Whitney forms provide the standard route to incorporate metric information into the discrete Hodge star operators, as described next.

4.2. Discrete Hodge Star Operator. In a source-free media, we can write the Hamiltonian as

$$
\mathscr{H}=\frac{1}{2} \int_{\Omega}(E \wedge D+H \wedge B)=\int_{\Omega}\left(E \wedge \star_{\epsilon} E+\star_{\mu^{-1}} B \wedge B\right) .
$$

Using (16), the lattice Hamiltonian assumes the expected quadratic form:

$$
\mathscr{H}=\sum_{i} \sum_{j} E_{i}\left[\star_{\epsilon}\right]_{i j} E_{j}+\sum_{i} \sum_{j} B_{i}\left[\star_{\mu^{-1}}\right]_{i j} B_{j},
$$

where we immediately identify the symmetric positive definite matrices:

$$
\begin{aligned}
& {\left[\star_{\epsilon}\right]_{i j}=\int_{\Omega} \omega_{i}^{1} \wedge{ }_{\epsilon} \omega_{j}^{1},} \\
& {\left[\star_{\mu^{-1}}\right]_{i j}=\int_{\Omega}\left(\star_{\mu^{-1}} \omega_{i}^{2}\right) \wedge \omega_{j}^{2}}
\end{aligned}
$$

as the discrete realization of the Hodge star operator(s) on a simplicial lattice $[23,69]$ so that

$$
\begin{gathered}
D_{i}=\sum_{j}\left[\star_{\epsilon}\right]_{i j} E_{j}, \\
H_{i}=\sum_{j}\left[\star_{\mu^{-1}}\right]_{i j} B_{j} .
\end{gathered}
$$

From the above, the Hamiltonian can be also expressed as

$$
\mathscr{H}=\sum_{i} E_{i} D_{i}+\sum_{i} H_{i} B_{i}
$$

4.3. Symplectic Structure and Dynamic Degrees of Freedom. The Hodge star matrices $\left[\star_{\epsilon}\right]$ and $\left[\star_{\mu^{-1}}\right]$ have different sizes. The number of elements in $\left[\star_{\epsilon}\right]$ is equal to $N_{1} \times N_{1}$, whereas the number of elements in $\left[\star_{\mu^{-1}}\right]$ is equal to $N_{2} \times N_{2}$. In other words, $\Theta(E)=\Theta(D) \neq \Theta(B)=\Theta(H)$, where $\Theta$ denotes the number of (discrete) degrees of freedom in the corresponding field.

One important property of a Hamiltonian system is its symplectic character, associated with area preservation in phase space. The symplectic character of the Hamiltonian in principle would require a canonical pair such as $E, B$ to have identical number of degrees of freedom. This apparent contradiction can be explained by the fact that Maxwell's equations (10) and (11) can be thought as aconstrained dynamic system (by the divergence conditions) so that, even though $\Theta(E) \neq \Theta(B)$, we still have $\Theta^{d}(E)=\Theta^{d}(B)$, where $\Theta^{d}$ denotes the number of dynamic degrees of freedom. This is discussed further below in Section 6, in connection with the discrete Hodge decomposition on a lattice.

\section{Semidiscrete Equations}

5.1. Local and Ultralocal Lattice Coupling. By using a contraction in the form of (2) on both sides of (10) with every face $\sigma_{2, j}$ of $\mathscr{K}$, and using the fact that $\left\langle\sigma_{2, j}, \omega_{i}^{2}\right\rangle=\left\langle\sigma_{1, j}, \omega_{i}^{1}\right\rangle=\delta_{i j}$ from (14), we get

$$
\begin{gathered}
\left\langle\sigma_{2, j}, \partial_{t} B\right\rangle=\partial_{t} \sum_{i} B_{i}\left\langle\sigma_{2, j}, \omega_{i}^{2}\right\rangle=\partial_{t} B_{j} \\
\left\langle\sigma_{2, j}, d E\right\rangle=\left\langle\partial \sigma_{2, j}, E\right\rangle=\sum_{i} E_{i} \sum_{k} C_{j k}^{1}\left\langle\sigma_{1, k}, \omega_{i}^{1}\right\rangle=\sum_{i} C_{j i}^{1} E_{i}
\end{gathered}
$$

so that

$$
-\partial_{t} B_{i}=\sum_{j} C_{i j}^{1} E_{j}
$$

where the index $i$ runs over all faces of the primal lattice. On the dual lattice $\widetilde{\mathscr{K}}$, we can similarly contract both sides of (11) with every dual face $\widetilde{\sigma}_{2, j}$ to get

$$
\partial_{t} D_{i}=\sum_{j} \widetilde{C}_{i j}^{1} H_{j}
$$

where now the index $i$ runs over all faces of the dual lattice. Using (20) and the fact that, in three-dimensions $\widetilde{C}_{i j}^{1}=C_{j i}^{1}$ [22] (up to possible boundary terms ignored here), we can write the last equation in terms of primal lattice quantities as

$$
\partial_{t} \sum_{j}\left[\star_{\epsilon}\right]_{i j} E_{j}=\sum_{j} C_{j i}^{1} \sum_{k}\left[\star_{\mu^{-1}}\right]_{j k} B_{k}
$$

or, by using the inverse Hodge star matrix $\left[\star_{\epsilon}\right]_{i j}^{-1}$, as

$$
\partial_{t} E_{i}=\sum_{j} \Upsilon_{i j} B_{j}
$$


with

$$
\Upsilon_{i j} \doteq \sum_{k} \sum_{l}\left[\star_{\epsilon}\right]_{i k}^{-1} C_{l k}^{1}\left[\star_{\mu^{-1}}\right]_{l j}
$$

The matrix $[Y]$ can be viewed as the discrete realization, for $p=2$, of the codifferential operator $\delta=(-1)^{p} *^{-1} d *$ that maps $p$-forms to $(n-p)$-forms [35].

Since the continuum operators $\star_{\epsilon}$ and $\star_{\mu^{-1}}$ are local [46] and, as seen, Whitney forms (13) have local support, it follows that the matrices $\left[\star_{\epsilon}\right]$ and $\left[\star_{\mu^{-1}}\right]$ are sparse, indicative of an ultralocal coupling (in the terminology of [70]). In contrast, the numerical inverse $\left[\star_{\epsilon}\right]^{-1}$ used in (27) is, in general, not sparse so that the field coupling between distant elements is nonzero. The lack of sparsity is a potential bottleneck in practical simulations. However, because the coupling strength in this case decays exponentially [29, 44], we can still say (using again the terminology of [70]) that the resulting discrete operator encoded by the matrix in (27) is local. In practical terms, the exponential decay allows one to set a cutoff on the nonzero elements of $\left[\star_{\epsilon}\right]$, based on element magnitudes or on the sparsity pattern of the original matrix $\left[\star_{\epsilon}\right]$, to build a sparse approximate inverse for $\left[\star_{\epsilon}\right]$ and hence recover back an ultralocal representation for $\star_{\epsilon}{ }^{-1}$ $[29,71]$. The sparsity pattern of $\left[\star_{\epsilon}\right]$ encodes the nearestneighbor edge information of the mesh and, consequently, the sparsity pattern of $\left[\star_{\epsilon}\right]^{k}$ likewise encodes successive " $k$ level" neighbors. The latter sparsity patterns can be used to build, quite efficiently, sparse approximations for $\left[\star_{\epsilon}\right]^{-1}$, as detailed in [29]. Once such sparse representations are obtained, (23) and (26) can be used in tandem to construct a marching-on-time algorithm (e.g., see Section 9.1 ahead) with a sparse structure and hence amenable for large-scale problems.

\subsection{Barycentric Dual and Barycentric Decomposition Lattices.} An alternative approach, aimed at constructing a sparse discrete Hodge star for $\star_{\epsilon^{-1}}$ directly from the dual lattice geometry is described in [27], based on earlier ideas exposed in $[24,72]$. This approach is based on the fact that both primal $\mathscr{K}$ and dual $\widetilde{\mathscr{K}}$ lattices can be decomposed into a third (underlying) lattice $\widehat{\mathscr{K}}$ by means of a barycentric decomposition, see [24]. The dual lattice $\widetilde{\mathscr{K}}$ in this case is called the barycentric dual lattice $[27,72]$ and the underlying lattice $\widehat{\mathscr{K}}$ is called the barycentric decomposition lattice. Importantly, $\widehat{\mathscr{K}}$ is simplicial and hence admits Whitney forms built on it using (13). Whitney forms on $\widehat{\mathscr{K}}$ can be used as building blocks to construct (dual) Whitney forms on the (nonsimplicial) $\widetilde{\mathscr{K}}$, and from that, a sparse inverse discrete Hodge star $\left[\star_{\epsilon^{-1}}\right]$ using integrals akin to (19). An explicit derivation of such dual lattice Whitney forms is provided in [73]. Furthermore, a recent comprehensive survey of this and other approaches based on dual lattices to construct discrete sparse inverse Hodge stars is provided in [74].
The barycentric dual lattice has the important property below associated with Whitney forms:

$$
\left\langle\widetilde{\sigma}_{(n-p), i}, \star \omega^{p}\left[\sigma_{p, j}\right]\right\rangle=\int_{\widetilde{\sigma}_{(n-p), i}} \star \omega^{p}\left[\sigma_{p, j}\right]=\delta_{i j},
$$

where $\star$ stands for the spatial Hodge star operator (distilled from constitutive material properties), and $\widetilde{\sigma}_{(n-p), i}$ is the dual element to $\sigma_{p, i}$ on the barycentric dual lattice. The operator $\star$ is such that

$$
\int_{\Omega} \omega^{p} \wedge \star \omega^{p}=\int_{\Omega}|\omega|^{2} d v
$$

where $|\omega|^{2}$ is the two-norm of $\omega^{p}$, and $d v$ is the volume element.

The identity (28) plays the role of structural property (14), on the dual lattice side. We stress that identity (28) is a distinctively characteristic feature of the barycentric dual lattice not shared by other geometrical constructions for the dual lattice. In other words, compatibility with Whitney forms via (28) naturally forces one to choose the dual lattice to be the barycentric dual.

From the above, one can also define a (Hodge) duality operator directly on the space of chains, that is, $\star_{K}: \Gamma_{p}(\mathscr{K}) \mapsto$ $\Gamma_{n-p}(\widetilde{\mathscr{K}})$ with $\star_{K}\left(\sigma_{p, i}\right)=\widetilde{\sigma}_{(n-p), i}$ and $\star_{\widetilde{K}}: \Gamma_{p}(\widetilde{\mathscr{K}}) \mapsto \Gamma_{n-p}(\mathscr{K})$ with $\star_{K}\left(\widetilde{\sigma}_{p, i}\right)=\widetilde{\sigma}_{(n-p), i}$, so that $\star_{K} \star_{\widetilde{K}}={ }{ }_{\widetilde{K}}{ }_{K}=1$. This construction is detailed in [24].

5.3. Galerkin Duality. Even though we have chosen to assign $E$ and $B$ to the primal (simplicial) lattice, and consequently $D, H, J$, and $\rho$ to the dual (nonsimplicial) lattice, the reverse is equally possible. In this case, the fields $D, H$ become associated to a simplicial lattice and hence can be expressed in terms of Whitney forms; the expressions dual to (16) are now

$$
\begin{gathered}
H=\sum_{i} H_{i} \omega_{i}^{1}, \\
D=\sum_{i} D_{i} \omega_{i}^{2}
\end{gathered}
$$

with sums running over primal edges and primal faces, respectively, and where

$$
\begin{aligned}
& E_{i}=\sum_{j}\left[\star_{\epsilon^{-1}}\right]_{i j} D_{j}, \\
& B_{i}=\sum_{j}\left[\star_{\mu}\right]_{i j} H_{j}
\end{aligned}
$$

with

$$
\begin{gathered}
{\left[\star_{\epsilon^{-1}}\right]_{i j}=\int_{\Omega}\left(\star_{\epsilon^{-1}} \omega_{i}^{2}\right) \wedge \omega_{j}^{2},} \\
{\left[\star_{\mu}\right]_{i j}=\int_{\Omega} \omega_{i}^{1} \wedge \star_{\mu} \omega_{j}^{1},}
\end{gathered}
$$

and the two Hodge star maps now used are such that, in the continuum, $\star_{\epsilon^{-1}}: \Lambda^{2}(\Omega) \rightarrow \Lambda^{1}(\Omega)$ and $\star_{\mu}: \Lambda^{1}(\Omega) \rightarrow$ 
$\Lambda^{2}(\Omega)$, and, on the lattice, $\left[\star_{\epsilon^{-1}}\right]: \Gamma^{2}(\mathscr{K}) \rightarrow \Gamma^{1}(\widetilde{\mathscr{K}})$ and $\left[\star_{\mu}\right]: \Gamma^{1}(\mathscr{K}) \rightarrow \Gamma^{2}(\widetilde{\mathscr{K}})$. This alternate choice entails a duality between these two formulations, dubbed "Galerkin duality." This is explored in more detail in [44].

\section{Discrete Hodge Decomposition and Euler's Formula}

For any $p$-form $\alpha^{p}$, we can write

$$
\alpha^{p}=d \zeta^{p-1}+\delta \beta^{p+1}+\chi^{p}
$$

where $\chi^{p}$ is a harmonic form [31]. This Hodge decomposition is unique. In the particular case of the 1 -form $E$, we have

$$
E=d \phi+\delta A+\chi
$$

where $\phi$ is a 0 -form and $A$ is a 2 -form, with $d \phi$ representing the static field, $\delta A$ the dynamic field, and $\chi$ the harmonic field component (if any). In a contractible domain, $\chi$ is identically zero and the Hodge decomposition simplifies to

$$
E=d \phi+\delta A,
$$

more usually known as Helmholtz decomposition in three dimensions.

In the discrete setting, the degrees of freedom of $\phi$ are associated to the nodes of the primal lattice. Likewise, the degrees of freedom of $A$ are associated to the facets of the primal lattice. Consequently, we have from (35) that

$$
\begin{aligned}
\Theta^{d}(E) & =N_{E}^{h}-N_{V}^{h} \\
& =\left(N_{E}-N_{E}^{b}\right)-\left(N_{V}-N_{V}^{b}\right) \\
& =N_{E}-N_{V},
\end{aligned}
$$

where $N_{V}$ is the number of primal nodes, $N_{E}$ the number of primal edges, and $N_{F}$ the number of primal facets, with superscript $b$ standing for boundary (fixed) elements and $h$ for interior (free) elements.

On the other hand, once we identify the lattice as a network of (in general) polyhedra, we can apply Euler's polyhedron formula on the primal lattice to obtain [44]

$$
N_{V}-N_{E}=1-N_{F}+N_{P}
$$

where $N_{P}$ represents the number of volume cells comprising the primal lattice. A similar Euler's polyhedron formula applies to the (closed, two-dimensional) boundary of the primal lattice:

$$
N_{V}^{b}-N_{E}^{b}=2-N_{F}^{b}
$$

Combining (37) and (38), we have

$$
\left(N_{E}-N_{E}^{b}\right)-\left(N_{V}-N_{V}^{b}\right)=\left(N_{F}-N_{F}^{b}\right)-\left(N_{P}-1\right) .
$$

From the Hodge decomposition (35), we see that $\Theta^{d}(E)$ is

$$
\begin{aligned}
\Theta^{d}(E) & =N_{E}^{i n}-N_{V}^{i n} \\
& =\left(N_{E}-N_{E}^{b}\right)-\left(N_{V}-N_{V}^{b}\right) .
\end{aligned}
$$

Note that the divergence free condition $d B=0$ produces one constraint on the 2 -form $B$ for each volume element. This constraint also spans the whole lattice boundary. The total number of the constrains for $B$ is, therefore, $\left(N_{P}-1\right)$. Consequently, we have

$$
\begin{aligned}
\Theta^{d}(B) & =N_{F}^{i n}-\left(N_{P}-1\right) \\
& =\left(N_{F}-N_{F}^{b}\right)-\left(N_{P}-1\right)
\end{aligned}
$$

so that

$$
\Theta^{d}(B)=\Theta^{d}(E) .
$$

This discussion can be generalized to lattices on noncontractible domains with any number of holes (genus), where the identity $\Theta^{d}(B)=\Theta^{d}(E)$ is also satisfied [31]. Moreover, from Hodge star isomorphism, we have $\Theta^{d}(D)=\Theta^{d}(E)$ and $\Theta^{d}(H)=\Theta^{d}(B)$.

In general, we can trace a direct correspondence between quantities in the Euler polyhedron formula to the quantities in the Hodge decomposition formula. For example, each term in the two-dimensional Euler's formula $N_{E}=N_{V}+\left(N_{F}-\right.$ $1)+g$ is associated to a corresponding term in $E=d \phi+$ $\delta A+\chi$, that is, the number of edges $N_{E}$ corresponds to the dimension of the space of lattice 1 -forms $E$, which is the sum of the number of nodes $N_{V}$ (dimension of the space of discrete 0 -forms $\phi$ ), the number of faces $\left(N_{F}-1\right)$ (dimension of the space of discrete 2-forms $A$ ), and the number of holes $g$ (dimension of the space of harmonic forms $\chi$ ). A similar correspondence can be traced on a three-dimensional lattice [31]. This correspondence provides a physical picture to Euler's formula and a geometric interpretation to the Hodge decomposition.

\section{Absorbing Boundary Conditions}

In many wave scattering simulations, the presence of longrange interactions with slow (algebraic) decay, together with practical limitations in computer memory resources, implies that open-space problems necessitate the use of special techniques to suppress finite-volume effects and emulate, for example, the Sommerfeld radiation condition at infinity. Perfectly matched layers (PML) are absorbing boundary conditions commonly used for this purpose [75-78]. In the continuum limit, the PML provides a reflectionless absorption of outgoing waves, in such a way that when the PML is used to truncate a computational lattice, finite-volume effects such as spurious reflections from the outer boundary are exponentially suppressed. When first introduced in the literature [75], the PML relied upon the use of matched artificial electric and magnetic conductivities in Maxwell's equations and of a splitting of each vector field component into two subcomponents. Because of this, the resulting fields inside the PML layer are rendered "non-Maxwellian." The PML concept was later shown to be equivalent in the Fourier domain $\left(\partial_{t} \rightarrow-i \omega\right)$ to a complex coordinate stretching of the coordinate space (or an analytic continuation to a complexvalued coordinate space) [76-78] and, as such, applicable to any linear wave phenomena. 
Inside the PML, the (local) spatial coordinate $\zeta$ along the outward normal direction to each lattice boundary point is complexified as

$$
\zeta \longrightarrow \widetilde{\zeta}=\int_{0}^{\zeta} s_{\zeta}\left(\zeta^{\prime}\right) d \zeta^{\prime}
$$

where $s_{\zeta}$ is the so-called complex stretching variable written as $s_{\zeta}(\zeta, \omega)=a_{\zeta}(\zeta)+i \Omega_{\zeta}(\zeta) / \omega$ with $a_{\zeta} \geq 1$ and $\Omega_{\zeta} \geq 0$ (profile functions). The first inequality ensures that evanescent waves will have a faster exponential decay in the PML region, and the second inequality ensures that propagating waves will decay exponentially along $\zeta$ inside the PML. As opposed to some other lattice truncation techniques, the PML preserves the locality of the underlying differential operators and hence retains the sparsity of the formulation.

For Maxwell's equations, the PML can also be affected by means of artificial material tensors (Maxwellian PML) [79]. In three dimensions, the Maxwellian PML can be represented as a media with anisotropic permittivity and permeability tensors exhibiting stratification along the normal to the boundary $S$ that parametrizes the lattice truncation boundary. The PML tensors properties depend on the local geometry via the two principal curvatures of $S$ [8082]. The boundary surface $S$ is assumed (constructed) as doubly differentiable with non negative radii of curvature, otherwise dynamic instabilities ensue during a marching-ontime evolution [83].

From (43), the PML also admits a straightforward interpretation as a complexification of the metric $[38,84]$. As a result, the use of differential forms readily unifies the Maxwellian and non-Maxwellian PML formulations because the metric is explicitly factored out into the Hodge star operators-any transformation the metric corresponds, dually, to a transformation on the Hodge star operators that can be mimicked by modified constitutive relations [37]. In the differential forms framework, the PML is obtained by a mapping on the Hodge star operators: $\star_{\epsilon} \rightarrow \widetilde{\star}_{\epsilon}$ and $\star_{\mu^{-1}} \rightarrow \widetilde{\star}_{\mu^{-1}}$ induced by the complexification of the metric. The resulting differential forms inside the PML, $\widetilde{E}, \widetilde{D}, \widetilde{H}$, and $\widetilde{B}$ therefore obey "modified" Hodge relations $\widetilde{D}=\widetilde{\star}_{\epsilon} \widetilde{E}$ and $\widetilde{B}=\widetilde{\star}_{\mu^{-1}} \widetilde{H}$, but identical premetric equations (10) and (11). In other words, (10) and (11) are invariant under the transformation $(43)[38,84]$.

\section{Implementation of Space Charge Effects}

In many applications related to plasma physics or electronic devices, it is necessary to include space charges (uncompensated charge effects) into lattice models of macroscopic Maxwell's equations. This is typically done by representing the charged plasma media using particle-in-cell (PIC) methods that track the individual particles on the lattice [85-87]. The field/charge interaction is then modeled by (i) interpolating lattice fields (cochains) to particle positions (gather step), (ii) advancing particle positions and velocities in time using equations of motion, and (iii) interpolating back charge densities and currents onto the lattice as cochains (scatter step). In general, the "particles" do not need to be actual individual particles, but can be a collection thereof (macroparticles). To put it simply, incorporation of space charges requires two extra steps during the field update in any marching-ontime algorithm, which transfer information from the instantaneous field distribution to the particle kinematic update and vice versa. Conventionally, this information transfer relies on spatial interpolations that often violates the charge continuity equation and, as a result, leads to spurious charge deposition on the lattice nodes. On regular lattices, this problem can be corrected, for example, using approaches that either subtract a static solution (charges) from the electric field solution (Boris/DADI correction) or directly subtract the residual error on the Gauss law (Langdon-Marder correction) at each time step [88]. On irregular lattices, additional degrees of freedom can be added as coupled elliptical constraints to produce an augmented Lagrange multiplier system [89]. All these approaches necessitate changes on the original equations, while still allowing for small violations on charge conservation. In contrast, Whitney forms provide a direct route to construct gather and scatter steps that satisfy charge conservation exactly even on unstructured lattices [90, 91], as explained next. To conform to the vast majority of the plasma and electronic devices literature, we once more restrict ourselves here to the $3+1$ setting, even though a four-dimensional analysis in Minkowski space would have provided a more succinct discussion.

For the gather step, Whitney forms can be used to directly compute (interpolate) the fields at any location from the knowledge of its cochain values, such as in (16), for example. For the scatter step, charge movement can be modeled as the Hodge-dual of the current 2-form $J$, that is, as the 1form $\star J$ which can be expanded in terms of Whitney 1-forms on the primal lattice. Here, $\star$ represents again the spatial Hodge star in three dimensions distilled from macroscopic constitutive properties. The Hodge-dual current associated to an individual point charge can be expressed as $\star J=$ $q v^{b}$, where $q$ is the charge value, $v$ is the associated velocity vector, and $b$ is the "flat" operator or index-lowering canonical isomorphism that maps a vector to a 1-form, given by the Euclidean metric. Similarly, point charges can be encoded as the Hodge-dual of the charge density 3 -form $\rho$, that is, as the 0 -form $\star \rho$, which can be expanded in terms of Whitney 0 -forms on the primal lattice. These two Whitney maps are linked in such a way that the rate of change on the value of the 0 -cochain representing $\star \rho$ at a node is associated to the presence of a 1-cochain representing $\star J$ along the edges that touch that particular node, leading to exact charge conservation at the discrete level. To show this, consider for simplicity the two-dimensional case of a point charge $q$ moving from point $x^{(s)}$ to point $x^{(f)}$ during a time interval $\tau$ inside a triangular cell with nodes $\sigma_{0,0}, \sigma_{0,1}$, and $\sigma_{0,2}$, or simply 0,1 , and 2 . At any point $x$ inside this cell, the 0 -form $\star \rho$ can be scattered to these three adjacent nodes via

$$
\star \rho=q \sum_{i=1}^{3}\left\langle x, \omega_{i}^{0}\right\rangle \omega_{i}^{0},
$$

where we are again using the short-hand $\omega^{0}\left[\sigma_{0, i}\right]=\omega_{i}^{0}$, and the brackets represent the pairing expressed by (1). In this 
case, $p=0$ and the pairing integral in (1) reduces to a function evaluation at a point. Since Whitney 0 -forms are equal to the barycentric coordinates associated of a given node, that is, $\left\langle x, \omega_{i}^{0}\right\rangle=\lambda_{i}(x)$, we have the scattered charge $q \lambda_{i}^{s} \doteq q \lambda_{i}\left(x^{(s)}\right)$ on node $i$ for a charge $q$ at $x^{(s)}$, and, similarly, the scattered charge $q \lambda_{i}^{f}$ on node $i$ for a charge $q$ at $x^{(f)}$. The rate of scattered charge variation on a given node $i$ is, therefore, equal to $\dot{q}\left(\lambda_{i}^{f}-\lambda_{i}^{s}\right)$, where $\dot{q}=q / \tau$.

During $\tau$, the particle travels through a path $\ell$ from $x^{(s)}$ to $x^{(f)}$, and the corresponding $\star J$ can be expanded as a sum of Whitney 1-forms $\omega_{i j}^{1}$ associated to the three adjacent edges $\overline{i j}=\overline{01}, \overline{12}, \overline{20}$, that is,

$$
\star J=\dot{q} \sum_{\overline{i j}}\left\langle\ell, \omega_{\overline{i j}}^{1}\right\rangle \omega_{i \bar{j}}^{1} .
$$

The coefficients $\left\langle\ell, \omega_{\overline{i j}}^{1}\right\rangle$ represent the (oriented) current flow along the associated oriented edge, that is, the cochain representation of $\star J$ along edge $\overline{i j}$. Using (13), the sum of the total current magnitude scattered along edges $\overline{01}$ and $\overline{20}$ that flows into node 0 is, therefore,

$$
\dot{q}\left(-\left\langle\ell, \omega \frac{1}{01}\right\rangle+\left\langle\ell, \omega \frac{1}{20}\right\rangle\right)=\dot{q} \int_{\ell}\left(-\omega \frac{1}{01}+\omega \frac{1}{20}\right) .
$$

Using $\omega_{i j}^{1}=\lambda_{i} d \lambda_{j}-\lambda_{j} d \lambda_{i}$ and $\lambda_{1}+\lambda_{2}+\lambda_{3}=1$, the above reduces to

$$
\dot{q} \int_{\ell} d \lambda_{0}=\dot{q}\left(\lambda_{0}^{f}-\lambda_{0}^{s}\right)
$$

which exactly matches the rate of scattered charge variation on node 0 obtained before. It is clear that similar equalities hold for nodes 1 and 2. More fundamentally, these equalities are a direct consequence of the structural property (15).

\section{Outline of Related Discretization Methods}

We outline below various discretization programs that rely, one way or another, on tenets exposed above. The delineation is informed mostly by applications related to electrodynamics. As expected, this delineation is not too sharp because the programs share much in common.

9.1. Finite-Difference Time-Domain Method. In cubical lattices, the (lowest-order) Whitney forms can be represented by means of a product of pulse and "rooftop" functions on the three Cartesian coordinates [92]. This choice, together with the use of low-order quadrature rules to compute the Hodge star integrals in (19), leads to diagonal matrices $\left[\star_{\epsilon}\right],\left[\star_{\mu^{-1}}\right]$, and, consequently, also diagonal $\left[\star_{\epsilon}\right]^{-1},\left[\star_{\mu^{-1}}\right]^{-1}$ and sparse $[Y]$ so that an ultralocal equation results for (26). In this fashion, one obtains a "matrix-free" algorithm where no linear algebra is needed during a marching-ontime solution for the fields. This prescription exactly recovers the Yee's scheme [50] that forms the basis for the celebrated finite-difference time-domain (FDTD) method (see [51, 93] and references therein). FDTD adopts the simplest explicit, energy-conserving (symplectic) time-discretization for (23) and (26), which can be constructed by staggering the electric and magnetic fields in time and replacing time derivatives by central differences. This results in the following "leap-frog" marching-on-time scheme:

$$
\begin{gathered}
B_{i}^{n+1 / 2}=B_{i}^{n-1 / 2}-\Delta t\left(\sum_{j} C_{i j}^{1} E_{j}^{n}\right), \\
E_{i}^{n+1}=E_{i}^{n}+\Delta t\left(\sum_{j} \Upsilon_{i j} B_{j}^{n+1 / 2}\right),
\end{gathered}
$$

where the superscript $n$ denotes the time-step index, and $\Delta t$ is the time increment (assumed uniform for simplicity). The staggering of the fields in both space and time is consistent with the presence of two staggered hypercubical spacetime lattices $[48,94]$ that can be viewed as prismatic extrusions, along the time coordinate, from the two (dual) staggered spatial lattices. The staggering in time also provides a $O\left(\Delta t^{2}\right)$ truncation error. Higher-order FDTD schemes with faster convergence to the continuum can be constructed by using less local approximations for the spatial derivatives (or equivalently less sparse $\left[\star_{\epsilon}\right]$ and $\left.[\Upsilon]\right)$ and/or for the time derivatives [95-97].

9.2. Finite-Integration Technique. The finite-integration technique (FIT) [98-100] is closely related to FDTD, with the main distinction being that in FIT the discretized equations are derived from the integral form of Maxwell's equations applied to every cell. Assuming piecewise constant fields over each cell, the latter is equivalent to applying the (discrete version) of the generalized Stokes' theorem to the cochains in (23) and (24). Another difference is that the incidence matrices and material (Hodge star) matrices are treated separately in FIT. In other words, metric-free and metricdependent parts of the equations are factorized a priori, in a manner akin to that exposed in Sections 3 and 4. Like FDTD, FIT is based on dual staggered lattices and, for cubical lattices, it turns out that the lowest-order FIT is algorithmically equivalent to the lowest-order FDTD. The spatial operators in FIT can all be viewed as discrete incarnations of the exterior derivative for the various $p$, and as such, the exact sequence property of the underlying de Rham complex is automatically enforced by construction [55]. Because of this, it could perhaps be claimed that FIT provides a more systematic route for generalizations to irregular lattices than Yee's FDTD. Historically, FIT generalizations to irregular lattices have relied on the use of either projection operators [101] or Whitney forms [102] to construct discrete versions of the Hodge star operators (or their procedural equivalents); however, these generalizations do not necessarily recover the specific form of the discrete Hodge matrix elements expressed in (19).

9.3. Cell Method. Another related discretization method, based on general principles originally put forth in [47-49], is the Cell method [103-108]. Even though this method does 
not rely on Whitney forms for constructing discrete Hodge star operators (other geometrically based constructions are instead used), it is nevertheless still based upon the use of "domain-integrated" discrete variables that conform to the notion of discrete differential forms or cochains of various degrees and, as such, it is naturally suited for irregular lattices. The Cell method also employs metric-free discrete operators that satisfy the exactness property of the de Rham complex and make explicit use of a dual lattice (but not necessarily barycentric) motivated by the notion of inner and outer orientations. The relationships between the various discrete operators and "domain-integrated" field quantities (cochains) in the Cell method are built into general classification diagrams referred to as "Tonti diagrams" that reproduce correct commuting diagram properties of the underlying operators $[47,48]$.

9.4. Mimetic Finite Differences. "Mimetic" finite-difference methods, originally developed for nonorthogonal hexahedral structured lattices ("tensor-product grids") and later extended for irregular and polyhedral lattices [109-118], also share many of the properties exposed above. The thrust here is towards the construction of discrete versions of the differential operators divergence, gradient, and curl of vector calculus having "compatible" (in the sense of the exactness property of the underlying de Rham complex) domains and ranges and such that the resulting discrete equations exactly satisfy discrete conservation laws. In three dimensions, this naturally leads to the definition of three "natural" operators and three "adjoint" operators that can be associated with exterior derivative $d$ and the codifferential $\delta$, respectively, for $p=1,2,3$ (although the exterior calculus terminology is often not used explicitly in this context). Metric aspects are not factored out into Hodge star operators because the latter do not appear explicitly in the formulation; instead, their procedural analogues are embedded into the definition of the discrete differential operators themselves through a properly defined set of discrete inner products for discrete scalar and vector fields. In mimetic finite differences, the discrete analogues of the codifferential operator $\delta$ are full matrices, and the matrix-free character of FDTD is lacking even on orthogonal lattices. In spite of that, an obvious advantage of mimetic finite differences versus conventional FDTD is that the former methodology provides a more natural extension to nonorthogonal and irregular lattices. Note that higher-order versions of mimetic finite differences also exist $[119,120]$.

\subsection{Compatible Discretizations and Finite-Element Exterior} Calculus. In recent years, much attention has been devoted to the development of "compatible discretizations," an umbrella term used to denote spatial discretizations of partial differential equations seeking to provide finite-element spaces that reproduce the exactness of the underlying de Rham complex (or the correct cohomology in topologically nontrivial domains) [121-126]. In this program, Whitney forms play a role of providing "conforming" vector-valued functional (finite-element) spaces of Sobolev type. Specifically, Whitney 1-forms recover the space of "Nedelec edge-elements" or curlconforming Sobolev space $\mathbf{H}($ curl, $\Omega$ ) [127] and Whitney 2forms recover the space of "Raviart-Thomas elements" or divconforming Sobolev space $\mathbf{H}(\operatorname{div}, \Omega)$ [128]. In this regard, a relatively new advance here has been the development of new finite-element spaces, beyond those provided by Whitney forms, based on the Koszul complex [129]. The latter is key for the stable discretization of elastodynamics, which had been an outstanding problem for many decades. An excellent first-hand summary of these advances is provided in [130]. Another recent comparable approach aimed at the stable discretization of elastodynamics using bundle-valued discrete differential forms is described in [131].

We should note that the link between stability conditions of some mixed finite-element methods [127] and the complex of Whitney forms has a long history in the context of electrodynamics. This link was first established in $[55,132]$, and further explored, for example, in $[18,19,21,23,32,36,61$, 133-136].

9.6. Discrete Exterior Calculus. The "discrete exterior calculus" (DEC) is another discretization program aimed at developing ab initio consistent discrete models to describe field theories [91, 137-141]. The main thrust of this program is not tied to any particular field theory but rather seeks to develop fundamental discrete tools (field variables, operators), amenable to tackle a whole gamut of theories (electrodynamics, fluid dynamics, elastodynamics, etc.). This discretization program recognizes the key role played by discrete differential forms as well as the need to define primal and dual cell complexes. There is a perceived focus on the use of circumcentric dual lattices as opposed to barycentric duals $[138,139]$ (even though the former does not admit a metric-free construction), and the program does not emphasize the role of Whitney forms (at least on its earlier stages). On the other hand, it recognizes the need to address group-valued differential forms, as well as the mathematical objects that exist on the dual-bundle space together with the associated operators (such as contractions and Lie derivatives), in connection to discrete problems in mechanics, optimal control, and computer vision/graphics [137]. A recent discussion on obstacles associated with some of the DEC underpinnings is provided in [142].

\section{Appendices}

\section{A. Differential Forms and Lattice Fermions}

Differential $p$-forms can be viewed as antisymmetric covariant tensor fields on rank $p$. Therefore, the ingredients discussed above are applicable to any antisymmetric tensor field theory, including non-Abelian gauge field theories and even topological field theories such as Chern-Simons theory [72]. However, for (Dirac) fermion fields the situation is different and, at first, it would seem unclear how differential forms could be used to describe spinors. Nevertheless, a useful connection can indeed be established $[1,16,143]$. To briefly 
address this point, we consider the lattice transcription of the (one-flavor) Dirac equation here.

Needless to say, the topic of lattice fermions is vast and we cannot do much justice to it here; we focus only on aspects that are more germane to main theme of this paper. In accordance to the related literature on lattice fermions, we work on Euclidean spacetime with $\hbar=c=1$ in this appendix, and adopt the repeated index summation convention with $\mu, \nu$ as coordinate indices, where $x$ is a point in fourdimensional space.

It is well known that fermion fields defy a lattice description with local coupling that gives the correct energy spectrum in the limit of zero lattice spacing and the correct chiral invariance [144]. This is formally stated by the no-go theorem of Nielsen-Ninomiya [145] and is associated to the well-known "fermion-doubling" problem [146]. A perhaps less known fact is that it is possible to arrive at a "geometrical" interpretation of the source of this difficulty by considering the "generalization" of the Dirac equation $\left(\gamma^{\mu} \partial_{\mu}+m\right) \psi(x)=0$ given by the Dirac-Kähler equation:

$$
(d-\delta) \Psi(x)=-m \Psi(x) .
$$

The square of the Dirac-Kähler operator can be viewed as the counterpart of the Dirac operator in the sense that

$$
(d-\delta)^{2}=-(d \delta+\delta d)=-\square
$$

recovers the Laplacian operator in the same fashion as the Dirac operator squared does, that is, $\left(\gamma^{\mu} \partial_{\mu}\right)^{2}=-\partial_{\mu} \partial^{\mu}=-\square$, where $\gamma^{\mu}$ represents Euclidean gamma matrices.

The Dirac-Kähler equation admits a direct transcription on the lattice because both the exterior derivative $d$ and the codifferential $\delta$ can be simply replaced by its lattice analogues, as discussed before. However, for the Dirac equation the analogy has to further involve the relationship between the 4component spinor field $\psi$ and the object $\Psi$. This relationship was first established in $[16,17]$ for hypercubic lattices and later extended to nonhypercubic lattices in [10, 147]. The analysis of $[16,17]$ has shown that $\Psi$ can be represented by a 16-component complex-valued inhomogeneous differential form:

$$
\Psi(x)=\sum_{p=0}^{4} \alpha^{p}(x),
$$

where $\alpha^{0}(x)$ is a (1-component) scalar function of position or 0 -form, $\alpha^{1}(x)=\alpha_{\mu}^{1}(x) d x^{\mu}$ is a (4-component) 1 -form, and likewise for $p=2,3,4$ representing 2-, 3-, and 4-forms with 6-, 4-, and 1-components, respectively. By employing the following Clifford algebra product:

$$
d x^{\mu} \vee d x^{\nu}=g^{\mu \nu}+d x^{\mu} \wedge d x^{\nu}
$$

as using the anticommutative property of the exterior product $\wedge$, we have

$$
d x^{\mu} \vee d x^{\nu}+d x^{\nu} \vee d x^{\mu}=2 g^{\mu \nu}
$$

which exactly matches the anticommutator result of the $\gamma^{\mu}$ matrices, $\gamma^{\mu} \gamma^{\nu}+\gamma^{\nu} \gamma^{\mu}=2 g^{\mu \nu}$. This suggests that $d x^{\mu}$ plays the role of the $\gamma^{\mu}$ matrix in the space of inhomogeneous differential forms with Clifford product [148], that is,

$$
\gamma^{\mu} \partial_{\mu} \longmapsto d x^{\mu} \vee \partial_{\mu}
$$

keeping in mind that while $\gamma^{\mu} \partial_{\mu}$ acts on spinors, $d x^{\mu} \vee$ $\partial_{\mu}=(d-\delta)$ acts on inhomogeneous differential forms. This analysis leads to a "geometrical" interpretation of the popular Kogut-Susskind staggered lattice fermions [149, 150] because the latter can be made identical to lattice DiracKähler fermions after a simple relabeling of variables [17].

The 16-component object $\Psi$ can be viewed as a $4 \times 4$ matrix that produces a fourfold degeneracy with respect to the Dirac equation for $\psi$. This degeneracy is actually not a problem in the continuum because there is a well-defined procedure to extract the 4-components of $\psi$ from those of $\Psi[16,17]$, whereby the 16 scalar equations encoded by (A.1) all reduce to the same copy of the four equations encoded by the standard Dirac equation. This procedure is performed by a set of "projection operators" that form a group $[16$, 151]. On the lattice, however, the operators $d$ and $\partial$, as well as * (which plays a role on the space of inhomogeneous differential forms $\Psi$ analogous to that of $\gamma^{5}$ on the space of spinors $\psi[152])$, behave in such a way that their action leads to lattice translations. This is because cochains with different $p$ necessarily live on different lattice elements and also because $*$ is a map between different lattice elements. As a consequence, the product operation of such "group" is not closed anymore. This nonclosure also stems from the fact that the lattice operators $d$ and $\delta$ do not satisfy Leibnitz's rule [148]. Because of this, the degeneracy of the Dirac equation on the lattice is present at a more fundamental level and is harder to extricate using the Dirac-Kähler description than the analogous degeneracy in the continuum. In this regard, a new approach to identify the extraneous degrees of freedom away from the continuum was recently described in [153]. In addition, a split-operator approach to solve Dirac equation based on the methods of characteristics that purports to avoid fermion doubling while maintaining chiral symmetry on the lattice was very recently put forth in [154]. This approach preserves the linearity of the dispersion relation by a splitting of the original problem into a series of one-dimensional problems and the use of a upwind scheme with a CourantFriedrichs-Lewy (CFL) number equal to one, which provides an exact time evolution (i.e., with no numerical dispersion effects) along each reduced one-dimensional problem. The main (practical) obstacle in this case is the need to use very small lattice elements.

\section{B. Classification of Inconsistencies in Naive Discretizations}

We provide below a rough classification scheme of inconsistencies arising from naive discretizations of the differential calculus on irregular lattices.

\section{(i) Premetric Inconsistencies of First Kind}

We call premetric inconsistencies of the first kind those that are related to the primal or dual lattices taken as separate 
objects and that occur when the discretization violates one or more properties of the continuum theory that is invariant under homeomorphisms-for example, conservations laws that relate a quantity on a region $S$ with an associated quantity on the boundary of the region, $\partial S$ (a topological invariant). Perhaps the most illustrative example is violation of "divergence-free" conditions caused by improper construction of incidence matrices, whereby the nilpotency of the (adjoint) boundary operator, $\partial \circ \partial=0$, is not observed. This implies, in a dual fashion, that the identity $d^{2}=0$ is violated [22]. Stated in another way, the exact sequence property of the underlying de Rham differential complex is violated [155]. In practical terms, this leads to the appearance spurious charges and/or spurious ("ghost") modes. As the classification suggests, these properties are not related to metric aspects of the lattice, but only to its "topological aspects," that is, on how discrete calculus operators are defined vis-à-vis the lattice element connectivity. In more mathematical terms, one can say that the structure of the (co)homology groups of the continuum manifold is not correctly captured by the cell complex (lattice). We stress again that, given any dual lattice construction, premetric inconsistencies of the first kind are associated to the primal or dual lattice taken separately, and not necessarily on how they intertwine.

\section{(ii) Premetric Inconsistencies of Second Kind}

The second type of premetric inconsistency is associated to the breaking of some discrete symmetry of the Lagrangian. In mathematical terms, this type of inconsistency can occur when the bijective correspondence between $p$-cells of the primal lattice and $(n-p)$-cells of the dual lattice (an expression of Poincaré duality at the level of cellular homology [156], up to boundary terms) is violated. This is typified by "nonreciprocal" constructions of derivative operators, where the boundary operator effecting the spatial derivation on the primal lattice $K$ is not the dual adjoint (or the incidence matrix transpose) of the boundary operator on the dual lattice $\mathscr{K}$, for example, the identity $\widetilde{C}_{i j}^{p}=C_{j i}^{n-1-p}$ (up to boundary terms) used to obtain (25) is violated. One basic consequence of this violation is that the resulting discrete equations break timereversal symmetry. Consequently, the numerical solutions will violate energy conservation and produce either artificial dissipation or late-time instabilities [22]. Many algorithms developed over the years for hyperbolic partial differential equations do indeed violate these properties: they are dissipative and cannot be used for long integration times [157, 158].

It should be noted at this point that lattice field theories invariably break Lorentz covariance and many of the continuum Lagrangian symmetries and, as a result, violate conservation laws (currents) by virtue of Noether's theorem. For example, angular momentum conservation does not hold exactly on the lattice because of the lack of continuous rotational symmetry (note that discrete rotational symmetries can still be present). However, this latter type of symmetry breaking is of a fundamentally different nature because it is "controllable," that is, their effect on the computed solutions is made arbitrarily small in the continuum limit. More importantly, discrete transcriptions of the Noether's theorem can be constructed for Lagrangian symmetries on a lattice [13, 159], to yield exact conservation laws of (properly defined) quantities such as discrete energy and discrete momentum [3].

\section{(iii) Hodge Star Inconsistencies}

In the third type of inconsistency, we include those that arise in connection with metric properties of the lattice. Because the metric is entirely encoded in the Hodge star operators $[22,42,160]$, such inconsistencies can be simply understood as inconsistencies on the construction of discrete Hodge star operators (or their procedural analogues). For example, it is not uncommon for naïve discretizations in irregular lattices to yield asymmetric discrete Hodge operators, as noted in $[161,162]$. Even if symmetry is observed, nonpositive definiteness might ensue that is often associated with portions of the lattice with highly skewed or obtuse cells [101]. Lack of either of these properties leads to unconditional instabilities that destroy marching-on-time solutions [22]. When very long integration times are needed, asymmetry in the discrete Hodge matrices can be a problem even if produced at the level of machine rounding-off errors.

\section{Acknowledgments}

The author thanks Weng Chew, Burkay Donderici, Bo He, and Joonshik Kim for discussions. The author also thanks the editorial board for the invitation to contribute with this paper.

\section{References}

[1] I. Montvay and G. Münster, Quantum Fields on a Lattice, Cambridge Monographs on Mathematical Physics, Cambridge University Press, Cambridge, UK, 1997.

[2] A. Zee, Quantum Field Theory in a Nutshell, Princeton University Press, Princeton, NJ, USA, 2003.

[3] W. C. Chew, "Electromagnetic field theory on a lattice," Journal of Applied Physics, vol. 75, no. 10, pp. 4843-4850, 1994.

[4] L. S. Martin and Y. Oono, "Physics-motivated numerical solvers for partial differential equations," Physical Review E, vol. 57, no. 4, pp. 4795-4810, 1998.

[5] M. A. H. Lopez, S. G. Garcia, A. R. Bretones, and R. G. Martin, "Simulation of the transient response of objects buried in dispersive media," in Ultrawideband Short-Pulse Electromagnetics, vol. 5, Kluwer Academic Press, Dordrecht, The Netherlands, 2000.

[6] F. L. Teixeira, "Time-domain finite-difference and finiteelement methods for Maxwell equations in complex media," IEEE Transactions on Antennas and Propagation, vol. 56, no. 8, part 1, pp. 2150-2166, 2008.

[7] N. H. Christ, R. Friedberg, and T. D. Lee, "Gauge theory on a random lattice," Nuclear Physics B, vol. 210, no. 3, pp. 310-336, 1982.

[8] J. E. Bolander and N. Sukumar, "Irregular lattice model for quasistatic crack propagation," Physical Review B, vol. 71, Article ID 094106, 2005.

[9] J. M. Drouffe and K. J. M. Moriarty, "U(2) four-dimensional simplicial lattice gauge theory," Zeitschrift für Physik C, vol. 24, no. 3, pp. 395-403, 1984. 
[10] M. Göckeler, "Dirac-Kähler fields and the lattice shape dependence of fermion flavour," Zeitschrift für Physik C, vol. 18, no. 4, pp. 323-326, 1983.

[11] J. Komorowski, "On finite-dimensional approximations of the exterior differential codifferential and Laplacian on a Riemannian manifold," Bulletin de l'Académie Polonaise des Sciences, vol. 23, no. 9, pp. 999-1005, 1975.

[12] J. Dodziuk, "Finite-difference approach to the Hodge theory of harmonic forms," American Journal of Mathematics, vol. 98, no. 1, pp. 79-104, 1976.

[13] R. Sorkin, "The electromagnetic field on a simplicial net," Journal of Mathematical Physics, vol. 16, no. 12, pp. 2432-2440, 1975.

[14] D. Weingarten, "Geometric formulation of electrodynamics and general relativity in discrete space-time," Journal of Mathematical Physics, vol. 18, no. 1, pp. 165-170, 1977.

[15] W. Müller, "Analytic torsion and R-torsion of Riemannian manifolds," Advances in Mathematics, vol. 28, no. 3, pp. 233-305, 1978.

[16] P. Becher and H. Joos, "The Dirac-Kähler equation and fermions on the lattice," Zeitschrift für Physik. C, vol. 15, no. 4, pp. 343-365, 1982.

[17] J. M. Rabin, "Homology theory of lattice fermion doubling," Nuclear Physics. B, vol. 201, no. 2, pp. 315-332, 1982.

[18] A. Bossavit, Computational Electromagnetism. Variational Formulations, Complementarity, Edge Elements, Electromagnetism, Academic Press, San Diego, Calif, USA, 1998.

[19] A. Bossavit, "Differential forms and the computation of fields and forces in electromagnetism," European Journal of Mechanics. B, vol. 10, no. 5, pp. 474-488, 1991.

[20] C. Mattiussi, "An analysis of finite volume, finite element, and finite difference methods using some concepts from algebraic topology," Journal of Computational Physics, vol. 133, no. 2, pp. 289-309, 1997.

[21] L. Kettunen, K. Forsman, and A. Bossavit, "Discrete spaces for div and curl-free fields," IEEE Transactions on Magnetics, vol. 34, pp. 2551-2554, 1998.

[22] F. L. Teixeira and W. C. Chew, "Lattice electromagnetic theory from a topological viewpoint," Journal of Mathematical Physics, vol. 40, no. 1, pp. 169-187, 1999.

[23] T. Tarhasaari, L. Kettunen, and A. Bossavit, "Some realizations of a discrete Hodge operator: a reinterpretation of finite element techniques," IEEE Transactions on Magnetics, vol. 35, no. 3, pp. 1494-1497, 1999.

[24] S. Sen, S. Sen, J. C. Sexton, and D. H. Adams, "Geometric discretization scheme applied to the abelian Chern-Simons theory," Physical Review E, vol. 61, no. 3, pp. 3174-3185, 2000.

[25] J. A. Chard and V. Shapiro, "A multivector data structure for differential forms and equations," Mathematics and Computers in Simulation, vol. 54, no. 1-3, pp. 33-64, 2000.

[26] P. W. Gross and P. R. Kotiuga, "Data structures for geometric and topological aspects of finite element algorithms," in Geometric Methods in Computational Electromagnetics, F. L. Teixeira, Ed., vol. 32 of Progress in Electromagnetics Research, pp. 151-169, EMW Publishing, Cambridge, Mass, USA, 2001.

[27] F. L. Teixeira, "Geometrical aspects of the simplicial discretization of Maxwell's equations," in Geometric Methods in Computational Electromagnetics, F. L. Teixeira, Ed., vol. 32 of Progress in Electromagnetics Research, pp. 171-188, EMW Publishing, Cambridge, Mass, USA, 2001.
[28] T. Tarhasaari and L. Kettunen, "Topological approach to computational electromagnetism," in Geometric Methods in Computational Electromagnetics, F. L. Teixeira, Ed., vol. 32 of Progress in Electromagnetics Research, pp. 189-206, EMW Publishing, Cambridge, Mass, USA, 2001.

[29] J. Kim and F. L. Teixeira, "Parallel and explicit finite-element time-domain method for Maxwell's equations," IEEE Transactions on Antennas and Propagation, vol. 59, no. 6, part 2, pp. 2350-2356, 2011.

[30] A. S. Schwarz, Topology for Physicists, vol. 308 of Grundlehren der Mathematischen Wissenschaften, Springer, Berlin, Germany, 1994.

[31] B. He and F. L. Teixeira, "On the degrees of freedom of lattice electrodynamics," Physics Letters A, vol. 336, no. 1, pp. 1-7, 2005.

[32] B. He and F. L. Teixeira, "Mixed E-B finite elements for solving 1D, 2-D, and 3-D time-harmonic Maxwell curl equations," IEEE Microwave and Wireless Components Letters, vol. 17, no. 5, pp. 313-315, 2007.

[33] H. Whitney, Geometric Integration Theory, Princeton University Press, Princeton, NJ, USA, 1957.

[34] C. W. Misner, K. S. Thorne, and J. A. Wheeler, Gravitation, W. H. Freeman, San Francisco, Calif, USA, 1973.

[35] G. A. Deschamps, "Electromagnetics and differential forms," Proceedings of the IEEE, vol. 69, pp. 676-696, 1982.

[36] P. R. Kotiuga, "Metric dependent aspects of inverse problems and functionals based on helicity," Journal of Applied Physics, vol. 73, no. 10, pp. 5437-5439, 1993.

[37] F. L. Teixeira and W. C. Chew, "Unified analysis of perfectly matched layers using differential forms," Microwave and Optical Technology Letters, vol. 20, no. 2, pp. 124-126, 1999.

[38] F. L. Teixeira and W. C. Chew, "Differential forms, metrics, and the reflectionless absorption of electromagnetic waves," Journal of Electromagnetic Waves and Applications, vol. 13, no. 5, pp. 665-686, 1999.

[39] F. L. Teixeira, "Differential form approach to the analysis of electromagnetic cloaking and masking," Microwave and Optical Technology Letters, vol. 49, no. 8, pp. 2051-2053, 2007.

[40] A. H. Guth, "Existence proof of a nonconfining phase in fourdimensional U(1) lattice gauge theory," Physical Review D, vol. 21, no. 8, pp. 2291-2307, 1980.

[41] A. Kheyfets and W. A. Miller, "The boundary of a boundary principle in field theories and the issue of austerity of the laws of physics," Journal of Mathematical Physics, vol. 32, no. 11, pp. 3168-3175, 1991.

[42] R. Hiptmair, "Discrete Hodge operators," Numerische Mathematik, vol. 90, no. 2, pp. 265-289, 2001.

[43] B. He and F. L. Teixeira, "Geometric finite element discretization of Maxwell equations in primal and dual spaces," Physics Letters A, vol. 349, no. 1-4, pp. 1-14, 2006.

[44] B. He and F. L. Teixeira, "Differential forms, Galerkin duality, and sparse inverse approximations in finite element solutions of Maxwell equations," IEEE Transactions on Antennas and Propagation, vol. 55, no. 5, pp. 1359-1368, 2007.

[45] B. Donderici and F. L. Teixeira, "Conformal perfectly matched layer for the mixed finite element time-domain method," IEEE Transactions on Antennas and Propagation, vol. 56, no. 4, pp. 1017-1026, 2008.

[46] W. L. Burke, Applied Differential Geometry, Cambridge University Press, Cambridge, UK, 1985.

[47] E. Tonti, "The reason for analogies between physical theories," Applied Mathematical Modelling, vol. 1, no. 1, pp. 37-50, 1976. 
[48] E. Tonti, "Finite formulation of the electromagnetic field," in Geometric Methods in Computational Electromagnetics, F. L. Teixeira, Ed., vol. 32 of Progress in Electromagnetics Research, pp. 1-44, EMW Publishing, Cambridge, Mass, USA, 2001.

[49] E. Tonti, "On the mathematical structure of a large class of physical theories," Rendiconti della Reale Accademia Nazionale dei Lincei, vol. 52, pp. 48-56, 1972.

[50] K. S. Yee, "Numerical solution of initial boundary value problems involving Maxwell'sequation is isotropic media," IEEE Transactions on Antennas and Propagation, vol. 14, no. 3, pp. 302-307, 1969.

[51] A. Taflove, Computational Electrodynamics, Artech House, Boston, Mass, USA, 1995.

[52] R. A. Nicolaides and X. Wu, "Covolume solutions of threedimensional div-curl equations," SIAM Journal on Numerical Analysis, vol. 34, no. 6, pp. 2195-2203, 1997.

[53] L. Codecasa, R. Specogna, and F. Trevisan, "Symmetric positivedefinite constitutive matrices for discrete eddy-current problems," IEEE Transactions on Magnetics, vol. 43, no. 2, pp. 510515, 2007.

[54] B. Auchmann and S. Kurz, "A geometrically defined discrete hodge operator on simplicial cells," IEEE Transactions on Magnetics, vol. 42, no. 4, pp. 643-646, 2006.

[55] A. Bossavit, "Whitney forms: a new class of finite elements for three-dimensional computations in electromagnetics," IEE Proceedings A, vol. 135, pp. 493-500, 1988.

[56] P. W. Gross and P. R. Kotiuga, Electromagnetic Theory and Computation: A Topological Approach, vol. 48 of Mathematical Sciences Research Institute Publications, Cambridge University Press, Cambridge, UK, 2004.

[57] A. Bossavit, "Discretization of electromagnetic problems: the "generalized finite differences" approach," in Handbook of Numerical Analysis, vol. 13, pp. 105-197, North-Holland Publishing, Amsterdam, The Netherlands, 2005.

[58] B. He, Compatible discretizations of Maxwell equations [Ph.D. thesis], The Ohio State University, Columbus, Ohio, USA, 2006.

[59] R. Hiptmair, "Higher order Whitney forms," in Geometric Methods in Computational Electromagnetics, F. L. Teixeira, Ed., vol. 32 of Progress in Electromagnetics Research, pp. 271-299, EMW Publishing, Cambridge, Mass, USA, 2001.

[60] F. Rapetti and A. Bossavit, "Whitney forms of higher degree," SIAM Journal on Numerical Analysis, vol. 47, no. 3, pp. 23692386, 2009.

[61] J. Kangas, T. Tarhasaari, and L. Kettunen, "Reading Whitney and finite elements with hindsight," IEEE Transactions on Magnetics, vol. 43, no. 4, pp. 1157-1160, 2007.

[62] A. Buffa, J. Rivas, G. Sangalli, and R. Vázquez, "Isogeometric discrete differential forms in three dimensions," SIAM Journal on Numerical Analysis, vol. 49, no. 2, pp. 818-844, 2011.

[63] A. Back and E. Sonnendrücker, "Spline discrete differential forms," in Proceedings of ESAIM, vol. 35, pp. 197-202, March 2012.

[64] S. Albeverio and B. Zegarliński, "Construction of convergent simplicial approximations of quantum fields on Riemannian manifolds," Communications in Mathematical Physics, vol. 132, no. 1, pp. 39-71, 1990.

[65] S. Albeverio and J. Schäfer, "Abelian Chern-Simons theory and linking numbers via oscillatory integrals," Journal of Mathematical Physics, vol. 36, no. 5, pp. 2157-2169, 1995.

[66] S. O. Wilson, "Cochain algebra on manifolds and convergence under refinement," Topology and Its Applications, vol. 154, no. 9, pp. 1898-1920, 2007.
[67] S. O. Wilson, "Differential forms, fluids, and finite models," Proceedings of the American Mathematical Society, vol. 139, no. 7, pp. 2597-2604, 2011.

[68] T. G. Halvorsen and T. M. Sørensen, "Simplicial gauge theory and quantum gauge theory simulation," Nuclear Physics B, vol. 854, no. 1, pp. 166-183, 2012.

[69] A. Bossavit, "Computational electromagnetism and geometry: (5) the " Galerkin Hodge," Journal of the Japan Society of Applied Electromagnetics, vol. 8, pp. 203-209, 2000.

[70] E. Katz and U. J. Wiese, "Lattice fluid dynamics from perfect discretizations of continuum flows," Physical Review E, vol. 58, pp. 5796-5807, 1998.

[71] B. He and F. L. Teixeira, "Sparse and explicit FETD via approximate inverse hodge (Mass) matrix," IEEE Microwave and Wireless Components Letters, vol. 16, no. 6, pp. 348-350, 2006.

[72] D. H. Adams, "A doubled discretization of abelian ChernSimons theory," Physical Review Letters, vol. 78, no. 22, pp. 41554158, 1997.

[73] A. Buffa and S. H. Christiansen, "A dual finite element complex on the barycentric refinement," Mathematics of Computation, vol. 76, no. 260, pp. 1743-1769, 2007.

[74] A. Gillette and C. Bajaj, "Dual formulations of mixed finite element methods with applications," Computer-Aided Design, vol. 43, pp. 1213-1221, 2011.

[75] J.-P. Berenger, "A perfectly matched layer for the absorption of electromagnetic waves," Journal of Computational Physics, vol. 114, no. 2, pp. 185-200, 1994.

[76] W. C. Chew and W. H. Weedon, "3D perfectly matched medium from modified Maxwell's equations with stretched coordinates," Microwave and Optical Technology Letters, vol. 7, no. 13, pp. 599604, 1994.

[77] F. L. Teixeira and W. C. Chew, "PML-FDTD in cylindrical and spherical grids," IEEE Microwave and Guided Wave Letters, vol. 7, no. 9, pp. 285-287, 1997.

[78] F. Collino and P. Monk, "The perfectly matched layer in curvilinear coordinates," SIAM Journal on Scientific Computing, vol. 19, no. 6, pp. 2061-2090, 1998.

[79] Z. S. Sacks, D. M. Kingsland, R. Lee, and J. F. Lee, "Perfectly matched anisotropic absorber for use as an absorbing boundary condition," IEEE Transactions on Antennas and Propagation, vol. 43, no. 12, pp. 1460-1463, 1995.

[80] F. L. Teixeira and W. C. Chew, "Systematic derivation of anisotropic PML absorbing media in cylindrical and spherical coordinates," IEEE Microwave and Guided Wave Letters, vol. 7, no. 11, pp. 371-373, 1997.

[81] F. L. Teixeira and W. C. Chew, "Analytical derivation of a conformal perfectly matched absorber for electromagnetic waves," Microwave and Optical Technology Letters, vol. 17, no. 4, pp. 231236, 1998.

[82] B. Donderici and F. L. Teixeira, "Conformal perfectly matched layer for the mixed finite element time-domain method," IEEE Transactions on Antennas and Propagation, vol. 56, no. 4, pp. 1017-1026, 2008.

[83] F. L. Teixeira and W. C. Chew, "On Causality and dynamic stability of perfectly matched layers for FDTD simulations," IEEE Transactions on Microwave Theory and Techniques, vol. 47, no. 63, pp. 775-785, 1999.

[84] F. L. Teixeira and W. C. Chew, "Complex space approach to perfectly matched layers: a review and some new developments," International Journal of Numerical Modelling, vol. 13, no. 5, pp. 441-455, 2000. 
[85] R. W. Hockney and J. W. Eastwood, Computer Simulation Using Particles, IOP Publishing, Bristol, UK, 1988.

[86] T. Z. Ezirkepov, "Exact charge conservation scheme for particlein-cell simularion with an arbitrary form-factor," Computer Physics Communications, vol. 135, pp. 144-153, 2001.

[87] Y. A. Omelchenko and H. Karimabadi, "Event-driven, hybrid particle-in-cell simulation: a new paradigm for multi-scale plasma modeling," Journal of Computational Physics, vol. 216, no. 1, pp. 153-178, 2006.

[88] P. J. Mardahl and J. P. Verboncoeur, "Charge conservation in electromagnetic PIC codes; spectral comparison of Boris/DADI and Langdon-Marder methods," Computer Physics Communications, vol. 106, no. 3, pp. 219-229, 1997.

[89] F. Assous, "A three-dimensional time domain electromagnetic particle-in-cell codeon unstructured grids," International Journal of Modelling and Simulation, vol. 29, no. 3, pp. 279-284, 2009.

[90] A. Candel, A. Kabel, L. Q. Lee et al., "State of the art in electromagnetic modeling for the compact linear collider," Journal of Physics: Conference Series, vol. 180, no. 1, Article ID 012004, 2009.

[91] J. Squire, H. Qin, and W. M. Tang, "Geometric integration of the Vlaslov-Maxwell system with a variational partcile-in-cell scheme," Physics of Plasmas, vol. 19, Article ID 084501, 2012.

[92] R. A. Chilton, H-, P- and T-refinement strategies for the finitedifference-time-domain (FDTD) method developed via finiteelement (FE) principles [Ph.D. thesis], The Ohio State University, Columbus, Ohio, USA, 2008.

[93] K. S. Yee and J. S. Chen, "The finite-difference time-domain (FDTD) and the finite-volume time-domain (FVTD) methods in solving Maxwell's equations," IEEE Transactions on Antennas and Propagation, vol. 45, no. 3, pp. 354-363, 1997.

[94] C. Mattiussi, "The geometry of time-stepping," in Geometric Methods in Computational Electromagnetics, F. L. Teixeira, Ed., vol. 32 of Progress in Electromagnetics Research, pp. 123-149, EMW Publishing, Cambridge, Mass, USA, 2001.

[95] J. Fang, Time domain finite difference computation for Maxwell equations [Ph.D. thesis], University of California, Berkeley, Calif, USA, 1989.

[96] Z. Xie, C.-H. Chan, and B. Zhang, "An explicit fourth-order staggered finite-difference time-domain method for Maxwell's equations," Journal of Computational and Applied Mathematics, vol. 147, no. 1, pp. 75-98, 2002.

[97] S. Wang and F. L. Teixeira, "Lattice models for large scale simulations of coherent wave scattering," Physical Review E, vol. 69, Article ID 016701, 2004.

[98] T. Weiland, "On the numerical solution of Maxwell's equations and applications in accelerator physics," Particle Accelerators, vol. 15, pp. 245-291, 1996.

[99] R. Schuhmann and T. Weiland, "Rigorous analysis of trapped modes in accelerating cavities," Physical Review Special TopicsAccelerators and Beams, vol. 3, no. 12, pp. 28-36, 2000.

[100] L. Codecasa, V. Minerva, and M. Politi, "Use of barycentric dual grids," IEEE Transactions on Magnetics, vol. 40, pp. 1414-1419, 2004.

[101] R. Schuhmann and T. Weiland, "Stability of the FDTD algorithm on nonorthogonal grids related to the spatial interpolation scheme," IEEE Transactions on Magnetics, vol. 34, no. 5, pp. 2751-2754, 1998.

[102] R. Schuhmann, P. Schmidt, and T. Weiland, "A new Whitneybased material operator for the finite-integration technique on triangular grids," IEEE Transactions on Magnetics, vol. 38, no. 2, pp. 409-412, 2002.

[103] M. Bullo, F. Dughiero, M. Guarnieri, and E. Tittonel, "Isotropic and anisotropic electrostatic field computation by means of the cell method," IEEE Transactions on Magnetics, vol. 40, no. 2, pp. 1013-1016, 2004.

[104] P. Alotto, A. De Cian, and G. Molinari, "A time-domain 3-D fullMaxwell solver based on the cell method," IEEE Transactions on Magnetics, vol. 42, no. 4, pp. 799-802, 2006.

[105] M. Bullo, F. Dughiero, M. Guarnieri, and E. Tittonel, "Nonlinear coupled thermo-electromagnetic problems with the cell method," IEEE Transactions on Magnetics, vol. 42, no. 4, pp. 991994, 2006.

[106] P. Alotto, M. Bullo, M. Guarnieri, and F. Moro, "A coupled thermo-electromagnetic formulation based on the cell method," IEEE Transactions on Magnetics, vol. 44, no. 6, pp. 702-705, 2008.

[107] P. Alotto, F. Freschi, and M. Repetto, "Multiphysics problems via the cell method: the role of tonti diagrams," IEEE Transactions on Magnetics, vol. 46, no. 8, pp. 2959-2962, 2010.

[108] L. Codecasa, R. Specogna, and F. Trevisan, "Discrete geometric formulation of admittance boundary conditions for frequency domain problems over tetrahedral dual grids," IEEE Transactions on Antennas and Propagation, vol. 60, pp. 3998-4002, 2012.

[109] M. Shashkov and S. Steinberg, "Support-operator finitedifference algorithms for general elliptic problems," Journal of Computational Physics, vol. 118, no. 1, pp. 131-151, 1995.

[110] J. M. Hyman and M. Shashkov, "Mimetic discretizations for Maxwell's equations," Journal of Computational Physics, vol. 151, no. 2, pp. 881-909, 1999.

[111] J. M. Hyman and M. Shashkov, "The orthogonal decomposition theorems for mimetic finite difference methods," SIAM Journal on Numerical Analysis, vol. 36, no. 3, pp. 788-818, 1999.

[112] J. M. Hyman and M. Shashkov, "Adjoint operators for the natural discretizations of the divergence, gradient and curl on logically rectangular grids," Applied Numerical Mathematics, vol. 25, no. 4, pp. 413-442, 1997.

[113] J. M. Hyman and M. Shashkov, "Mimetic finite difference methods for Maxwell's equations and the equations of magnetic diffusion," in Geometric Methods in Computational Electromagnetics, F. L. Teixeira, Ed., vol. 32 of Progress in Electromagnetics Research, pp. 89-121, EMW Publishing, Cambridge, Mass, USA, 2001.

[114] J. Castillo and T. McGuinness, "Steady state diffusion problems on non-trivial domains: support operator method integrated with direct optimized grid generation," Applied Numerical Mathematics, vol. 40, no. 1-2, pp. 207-218, 2002.

[115] K. Lipnikov, M. Shashkov, and D. Svyatskiy, "The mimetic finite difference discretization of diffusion problem on unstructured polyhedral meshes," Journal of Computational Physics, vol. 211, no. 2, pp. 473-491, 2006.

[116] F. Brezzi and A. Buffa, "Innovative mimetic discretizations for electromagnetic problems," Journal of Computational and Applied Mathematics, vol. 234, no. 6, pp. 1980-1987, 2010.

[117] N. Robidoux and S. Steinberg, "A discrete vector calculus in tensor grids," Computational Methods in Applied Mathematics, vol. 11, no. 1, pp. 23-66, 2011.

[118] K. Lipnikov, G. Manzini, F. Brezzi, and A. Buffa, "The mimetic finite difference method for the 3D magnetostatic field problems on polyhedral meshes," Journal of Computational Physics, vol. 230, no. 2, pp. 305-328, 2011. 
[119] V. Subramanian and J. B. Perot, "Higher-order mimetic methods for unstructured meshes," Journal of Computational Physics, vol. 219, no. 1, pp. 68-85, 2006.

[120] L. Beirão da Veiga and G. Manzini, "A higher-order formulation of the mimetic finite difference method," SIAM Journal on Scientific Computing, vol. 31, no. 1, pp. 732-760, 2008.

[121] D. N. Arnold, "Differential complexes and numerical stability," in Proceedings of the International Congress of Mathematicians, vol. 1 of Plenary Lectures, pp. 137-157, Beijing, China, 2002.

[122] D. N. Arnold, P. B. Bochev, R. B. Lehoucq, R. A. Nicolaides, and M. Shashkov, Eds., Compatible Spatial Discretizations, vol. 142 of The IMA Volumes in Mathematics and Its Applications, Springer, New York, NY, USA, 2006.

[123] D. A. White, J. M. Koning, and R. N. Rieben, "Development and application of compatible discretizations of Maxwell's equations," in Compatible Spatial Discretizations, vol. 142 of The IMA Volumes in Mathematics and Its Applications, pp. 209-234, Springer, New York, NY, USA, 2006.

[124] P. Bochev and M. Gunzburger, "Compatible discretizations of second-order elliptic problems," Journal of Mathematical Sciences, vol. 136, no. 2, pp. 3691-3705, 2006.

[125] D. Boffi, "Approximation of eigenvalues in mixed form, discrete compactness property, and application to $h p$ mixed finite elements," Computer Methods in Applied Mechanics and Engineering, vol. 196, no. 37-40, pp. 3672-3681, 2007.

[126] P. Bochev, H. C. Edwards, R. C. Kirby, K. Peterson, and D. Ridzal, "Solving PDEs with Intrepid," Scientific Programming, vol. 20, pp. 151-180, 2012.

[127] J.-C. Nédélec, "Mixed finite elements in $\mathbf{R}^{3}$," Numerische Mathematik, vol. 35, no. 3, pp. 315-341, 1980.

[128] R. Hiptmair, "Canonical construction of finite elements," Mathematics of Computation, vol. 68, no. 228, pp. 1325-1346, 1999.

[129] V. W. Guillemin and S. Sternberg, Supersymmetry and Equivariant de Rham Theory, Mathematics Past and Present, Springer, Berlin, Germany, 1999.

[130] D. N. Arnold, R. S. Falk, and R. Winther, "Finite element exterior calculus, homological techniques, and applications," Acta Numerica, vol. 15, pp. 1-155, 2006.

[131] A. Yavari, "On geometric discretization of elasticity," Journal of Mathematical Physics, vol. 49, no. 2, article 022901, 2008.

[132] A. Bossavit, "Mixed finite elements and the complex of Whitney forms," in The Mathematics of Finite Elements and Applications, VI (Uxbridge, 1987), J. R. Whiteman, Ed., pp. 137-144, Academic Press, London, UK, 1988.

[133] M. F. Wong, O. Picon, and V. F. Hanna, "Finite element method based on Whitney forms to solve Maxwell equations in the time domain," IEEE Transactions on Magnetics, vol. 31, no. 3, pp. 1618-1621, 1995.

[134] M. Feliziani and F. Maradei, "Mixed finite-difference/Whitneyelements time-domain (FD/WE-TD) method," IEEE Transactions on Magnetics, vol. 34, no. 5, pp. 3222-3227, 1998.

[135] P. Castillo, J. Koning, R. Rieben, and D. White, "A discrete differential forms framework for computational electromagnetism," Computer Modeling in Engineering \& Sciences, vol. 5, no. 4, pp. 331-345, 2004.

[136] R. N. Rieben, G. H. Rodrigue, and D. A. White, "A high order mixed vector finite element method for solving the time dependent Maxwell equations on unstructured grids," Journal of Computational Physics, vol. 204, no. 2, pp. 490-519, 2005.

[137] M. Dsebrun, A. N. Hirani, and J. E. Mardsen, "Discrete exterior calculus for variational problem in computer vision and graphics," in Proceedings of the 42nd IEEE Conference on Decision and Control, pp. 4902-4907, Maui, Hawaii, USA, 2003.

[138] A. N. Hirani, Discrete exterior calculus [Ph.D. thesis], California Institute of Technology, Pasadena, Calif, USA, 2003.

[139] M. Desbrun, A. N. Hirani, M. Leok, and J. E. Mardsen, "Discrete exterior calculus," 2005, http://arxiv.org/abs/math/0508341.

[140] A. Gillette, "Notes on discrete exterior calculus," Tech. Rep., University of Texas at Austin, Austin, Texas, USA, 2009.

[141] J. B. Perot, "Discrete conservation properties of unstructures mesh schemes," Annual Review of Fluid Mechanics, vol. 43, pp. 299-318, 2011.

[142] P. R. Kotiuga, "Theoretical limitation of discrete exterior calculus in the context of computational electromagnetics," IEEE Transactions on Magnetics, vol. 44, pp. 1162-1165, 2008.

[143] W. Graf, "Differential forms as spinors," Annales de l'Institut Henri Poincaré A. Physique Théorique, vol. 29, no. 1, pp. 85-109, 1978.

[144] D. H. Adams, "Fourth root prescription for dynamical staggered fermions," Physical Review D, vol. 72, Article ID 114512, 2005.

[145] D. Friedan, "A proof of the Nielsen-Ninomiya theorem," Communications in Mathematical Physics, vol. 85, no. 4, pp. 481-490, 1982.

[146] I. F. Herbut, "Time reversal, fermion doubling, and the masses of lattice Dirac fermions in three dimensions," Physical Review B, vol. 83, no. 24, Article ID 245445, 2011.

[147] H. Raszillier, "Lattice degeneracies of fermions," Journal of Mathematical Physics, vol. 25, no. 6, pp. 1682-1693, 1984.

[148] I. Kanamori and N. Kawamoto, "Dirac-Kähler femion with noncommutative differential forms on a lattice," Nuclear Physics B-Proceedings Supplements, vol. 129, pp. 877-879, 2004.

[149] L. Susskind, "Lattice fermions," Physical Review D, vol. 16, no. 10, pp. 3031-3039, 1977.

[150] M. G. do Amaral, M. Kischinhevsky, C. A. A. de Carvalho, and F. L. Teixeira, "An efficient method to calculate field theories with dynamical fermions," International Journal of Modern Physics C, vol. 2, no. 2, pp. 561-600, 1991.

[151] I. M. Benn and R. W. Tucker, "The Dirac equation in exterior form," Communications in Mathematical Physics, vol. 98, no. 1, pp. 53-63, 1985.

[152] V. de Beauce, S. Sen, and J. C. Sexton, "Chiral dirac fermions on the latice using geometric discretization," Nuclear Physics BProceedings Supplements, vol. 129, pp. 468-470, 2004.

[153] D. H. Adams, "Theoretical foundation for the index theorem on the lattice with staggered fermions," Physical Review Letters, vol. 104, Article ID 141602, 2010.

[154] F. Fillion-Gourdeau, E. Lorin, and A. D. Bandrauk, "Numerical solution of the time-dependent Dirac equation in coordinate space without fermion-doubling," Computer Physics Communications, vol. 183, no. 7, pp. 1403-1415, 2012.

[155] D. N. Arnold, "Differential complexes and numerical stability," in Proceedings of the International Congress of Mathematicians, vol. 1 of Plenary Lecture, pp. 137-157, Higher Ed. Press, Beijing, China, 2002.

[156] J. R. Munkres, Topology, Pearson, 2nd edition, 2000.

[157] M. W. Chevalier, R. J. Luebbers, and V. P. Cable, "FDTD local grid with material traverse," IEEE Transactions on Antennas and Propagation, vol. 45, pp. 411-421, 1997.

[158] M. J. White, Z. Yun, and M. F. Iskander, "A new 3-D FDTD multigrid technique with dielectric traverse capabilities," IEEE Transactions on Microwave Theory and Techniques, vol. 49, no. 3, pp. 422-430, 2001. 
[159] S. H. Christiansen and T. G. Halvorsen, "A simplicial gauge theory," Journal of Mathematical Physics, vol. 53, no. 3, Article ID 033501, 17 pages, 2012.

[160] A. Bossavit, "Generalized finite differences'in computational electromagnetics," in Geometric Methods for Computational Electromagnetics, F. L. Teixeira, Ed., vol. 32 of Progress in Electromagnetics Research, pp. 45-64, EMW Publishing, Cambridge, Mass, USA, 2001.

[161] P. Thoma and T. Weiland, "A consistent subgridding scheme for the finite difference time domain method," International Journal of Numerical Modelling, vol. 9, no. 5, pp. 359-374, 1996.

[162] K. M. Krishnaiah and C. J. Railton, "Passive equivalent circuit of FDTD: an application to subgridding," Electronics Letters, vol. 33, no. 15, pp. 1277-1278, 1997. 


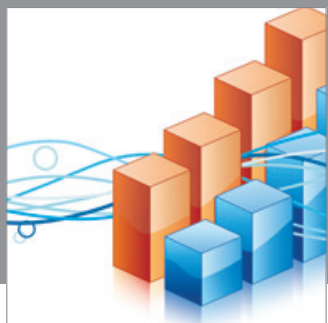

Advances in

Operations Research

mansans

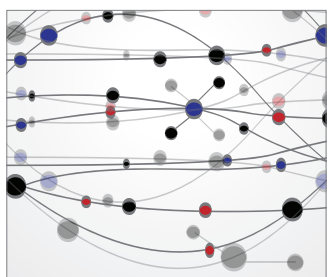

The Scientific World Journal
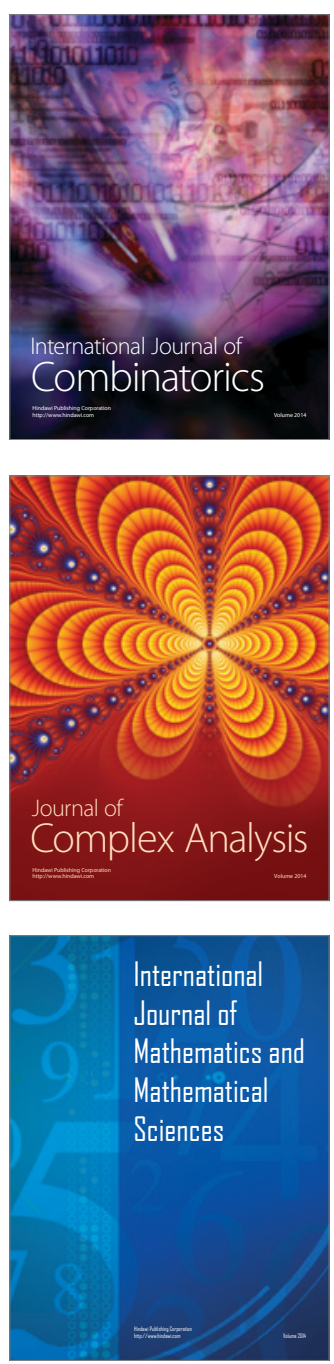
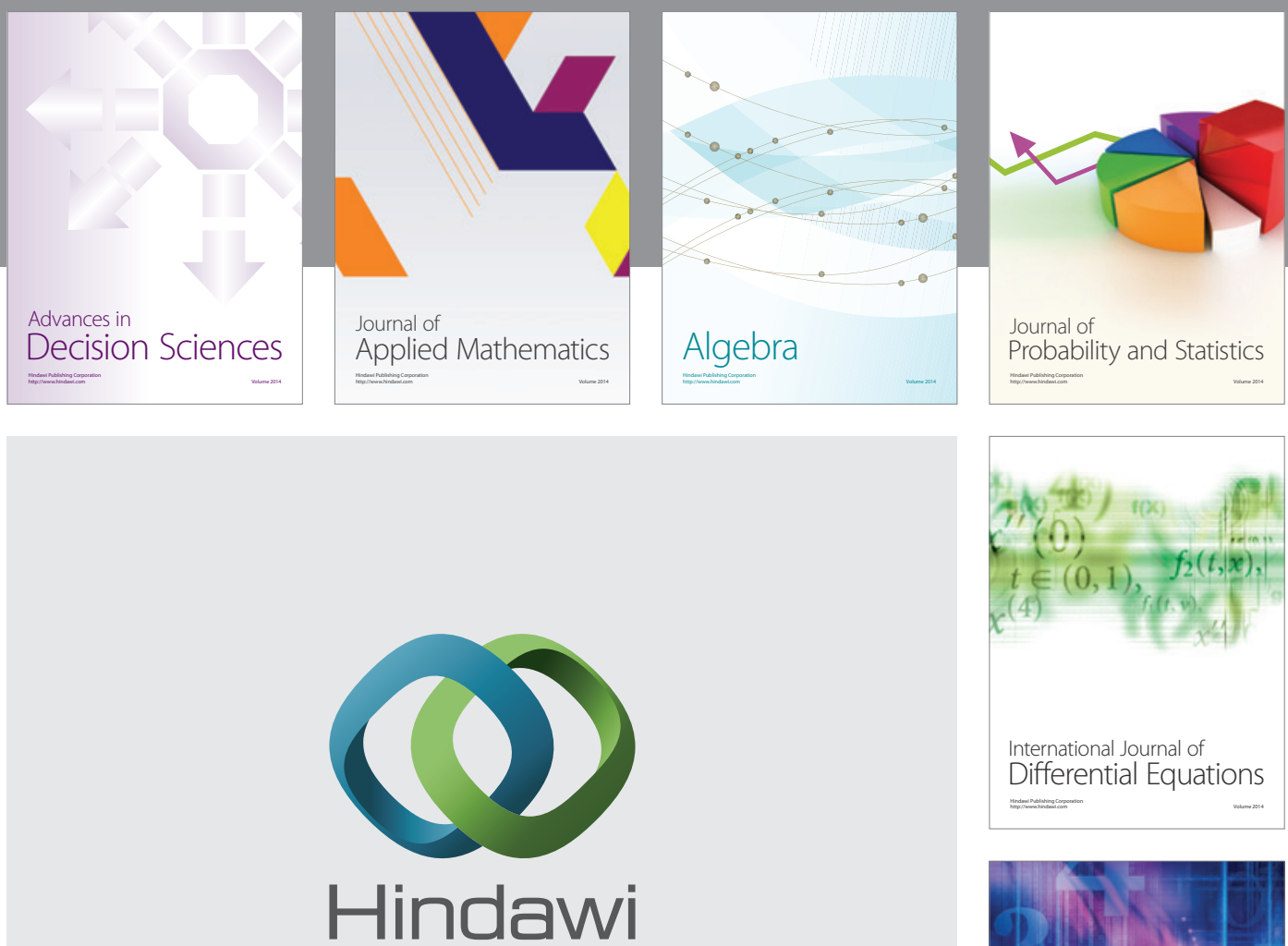

Submit your manuscripts at http://www.hindawi.com
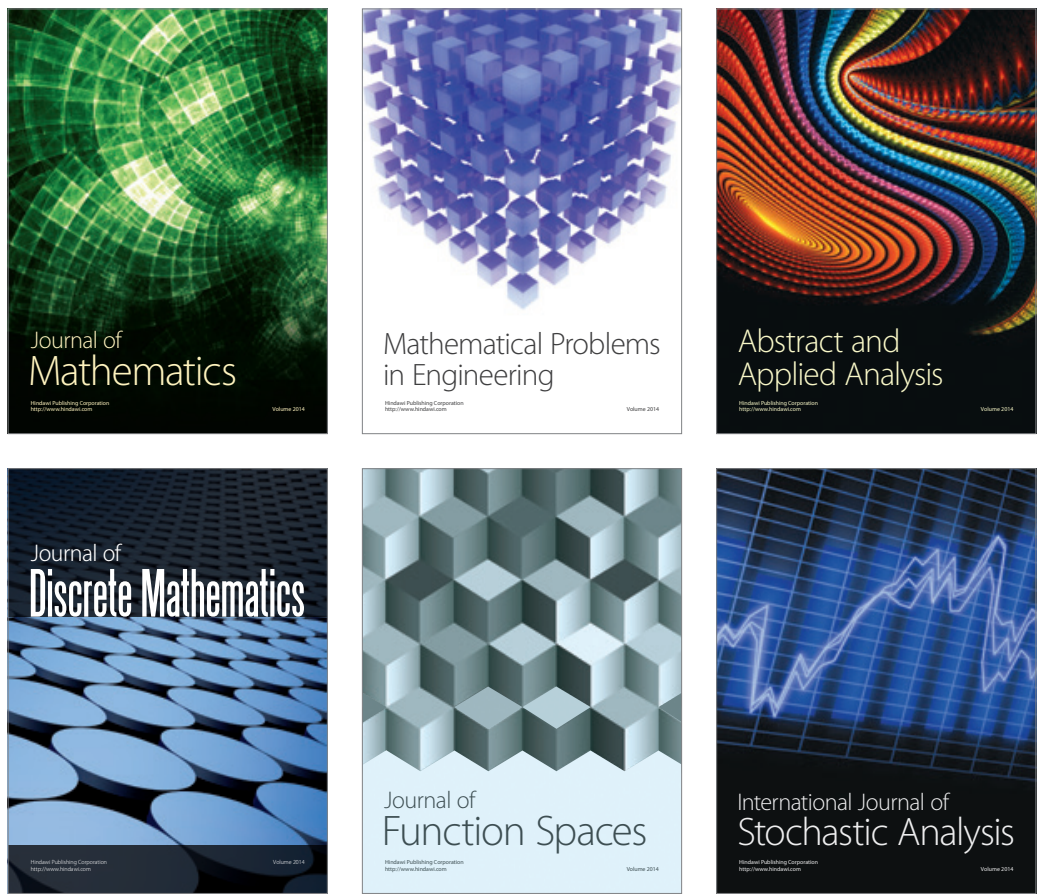

Journal of

Function Spaces

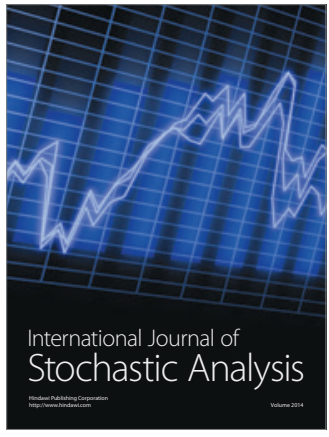

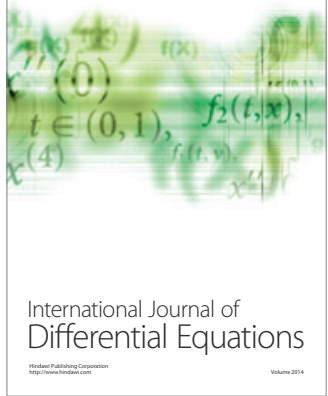
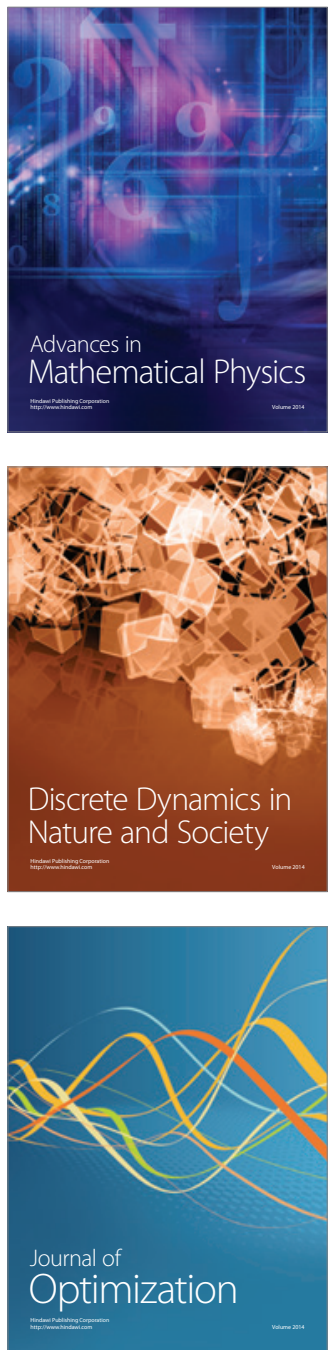